\title{
From wave function to crystal morphology: application to urea and alpha-glycine
}

\author{
E.S. Boek, D. Feil, W.J. Briels \\ Chemical Physics Laboratory, Uniwersity of Twente, P.O. Box 217, 7500 AE Enschede, Netherlands
}

and

\section{P. Bennema}

RIM Laboratory of Solid State Chemistry, Faculty of Science, University of Nijmegen, Toernooiveld, 6525 ED Nijmegen, Netherlands

Received 20 March 1991

\begin{abstract}
In this paper the relation between the molecular electron density distribution and the crystal growth morphology is investigated. Accurate charge densities derived from ab initio quantum chemical calculations were partitioned into multipole moments, to calculate the electrostatic contribution to the intermolecular interaction energy. For urea and $\alpha$-glycine the F-faces or connected nets were determined according to the Hartman-Perdok PBC theory. From attachment energy and critical Ising temperature calculations, theoretical growth forms were constructed using different atom-atom potential models. These were compared to the Donnay-Harker model, equilibrium form and experimental growth forms. In the case of $\alpha$-glycine, the theoretical growth forms are in good agreement with crystals grown from aqueous solution. Crystals obtained by sublimation seem to show some faces which are not F-faces sensu stricto.
\end{abstract}

\section{Introduction}

In recent years many papers have been written on the relation between crystal structure and morphology. Almost all of these are based on the pioneering work of Hartman and Perdok [2]. By means of their periodic bond chain (PBC) theory, crystal faces can be divided into slowly growing $\mathrm{F}$-faces and rapidly growing $\mathrm{S}$ - or $\mathrm{K}$-faces. This theory explains crystal morphology from the crystal structure quite well in a qualitative way. Taking intermolecular interaction energies into account, also a quantitative prediction of the kinetic growth form became possible: Hartman and Bennema [4] introduced the proportionality relation between the growth rate of an F-face and its attachment energy. A few years later Rijpkema et al. [8] developed an alternative theory, in which F-faces are regarded as connected nets for which critical roughening temperatures can be calculated in a two-dimensional Ising model. In both theories the interaction energy between elementary growth units plays a fundamental role. In molecular crystals containing molecules with strongly polar groups, the Coulomb energy is far more important than the Van der Waals energy. Therefore, the crystal habit is mainly determined by the charge distribution in the molecule. Berkovitch-Yellin [5] presented a successful method to derive growth forms for such crystals, using experimental electrostatic parameters from $\mathrm{X}$-ray diffraction data. Her work is based on the calculation of attachment energies without, however, taking PBCs and F-faces explicitly into account ("cheapest cut of slices through the crystal structure").

The present paper investigates if it is justified to refrain from such a structural PBC analysis. At 
first an overview is given of the concepts of different morphological theories. Secondly the intermolecular interaction is discussed. The Coulomb term is calculated from electrostatic multipoles which are derived from experimental as well as from theoretical charge distributions by means of stockholder partitioning; theoretical charge distributions seem to resemble experimental ones quite well [23] and, moreover, they can be obtained with less effort. Finally, these theories are applied to $\alpha$-glycine and urea. By comparing theoretical habits from different models for the atom-atom potential and the crystal morphology to experimental growth forms, it is investigated which model gives the best fit to the experiment.

\section{Concepts: morphological theories}

The first attempts to relate crystal morphology to internal structure at the molecular level were performed by Donnay and Harker [1]. They developed a purely geometrical law by stating that the growth rate of a crystal face increases with decreasing interplanar lattice spacing $d_{h k l}$. Submultiples of $d_{h k l}$ should be taken into account when translational elements like glide planes or screw axes are present perpendicular to the plane or when lattice centring occurs. In many cases this law describes the morphology quite well; violations are mostly due to pseudosymmetry effects, causing pseudoperiods of the surface structure. By means of their periodic bond chain (PBC) theory, Hartman and Perdok [2] developed a more physical method to derive slowly growing and thus habit-determining faces from the crystal structure. In this theory the crystal growth mechanism is regarded as the formation of strong bonds between crystallizing units (atoms, ions or molecules), so only nearest neighbours are considered. The procedure of a PBC analysis is as follows: first the strong bonds in the first coordination sphere of a reference unit are determined from the crystal structure. Next, PBCs (uninterrupted chains of bonds) in the direction [uvw] may be found from the set of strong bonds. These PBCs must be stoichiometric and have no dipole moment perpendicular to $[u \iota w]$. Then, new PBCs may be identified from a structure projection along the PBC direction [uvw]. When PBCs in the directions $[u v w]_{1}$ and $[u v w]_{2}$ are mutually connected within a slice $d_{h k l}$ such that $[u l w]_{1} \times$ $[u \iota w]_{2}=(h k l)$, the face is flat or $\mathrm{F}$.

Generally the $\mathrm{PBC}$ concept leads to three classes of crystal faces [3]:

F-faces (flat): containing at least two mutually connected PBCs in different directions within a slice $d_{h k l}$;

S-faces (stepped): containing only one PBC within $d_{h k i}$

K-faces (kinked): containing no PBC at all.

The three types of faces have different growth mechanisms: F-faces are considered to grow according to a layer mechanism by means of two-dimensional nucleation or spiral growth. Therefore they grow slowly and generally determine the crystal habit. S-faces need one-dimensional nucleation, while K-faces need no nucleation at all: therefore they grow quickly and are generally absent from the growth form.

By means of this formalism a qualitative prediction of the crystal habit has been made possible. For a quantitative approach the relative growth rates $R_{h k l}$ of the F-faces have to be known. Hartman and Bennema [4] showed that the growth rate $R_{h k l}$ can be approximated by putting it directly proportional to the attachment energy $E_{\text {all }}$, defined as the energy released per molecule when a slice with thickness $d_{h k l}$ crystallizes on an already existing crystal face. This energy is related to the crystal energy $E_{\text {cr }}$ by:

$E_{\mathrm{cr}}=E_{\mathrm{stice}}+E_{\mathrm{att}}$,

in which the slice energy $E_{\text {slice }}$ is defined as the energy released per molecule when a new slice $d_{h k l}$ is formed from the vapour. The crystal energy $E_{\mathrm{cr}}$ is defined as the energy per molecule released when the crystal is formed from the crystallizing particles. Therefore $E_{\mathrm{cr}}$ is the half crystal energy which is equal to the experimentally measurable sublimation energy. In general it can be said that the growth rate $R_{h k^{\prime}}$ is an increasing function of $E_{\text {att }}$. It might, however, be dangerous to apply the proportionality relation between $R_{h k l}$ and $E_{\text {att }}$ to all faces without per- 
forming a structural PBC analysis; S- or even $\mathrm{K}$-faces may have rather large negative slice energies (as will be shown in the case of $\alpha$-glycine) but as they do not grow layerwise and hence quickly, their occurrence on the theoretical growth form is fundamentally impossible.

In the present paper, crystal, slice and attachment energies of urea and $\alpha$-glycine were calculated by summing the intermolecular interactions between a reference molecule and all molecules in a crystal block around the reference molecule. For this purpose the program MOLINT was written. The size of the crystal block was determined by a summation limit of $25-30 \AA$, ensuring convergence of the electrostatic lattice sum [6]. To avoid dependence of the result on the choice of the reference molecule, the calculations were carried out over all unique molecules in the unit cell and the respective values were averaged. The slice energy $E_{\text {slice }}^{*}$, equal to twice $E_{\text {slice }}$ as defined in eq. (1), was calculated as the sum of the interactions between a reference molecule and all neighbours within a slice $d_{l k l}$, satisfying the condition:

$\left|h \cdot x_{j}+k \cdot y_{j}+l \cdot z_{j}\right|<0.5$,

in which $x, y$ and $z$ are the components of the vector from the centre of mass of the reference molecule to that of a molecule $j$ in the layer. Similarly, $E_{\text {att }}$ was determined by the condition:

$\left(h \cdot x_{j}+k \cdot y_{j}+l \cdot z_{j}\right)>0.5$.

A second method which predicts crystal morphology from statistical mechanics was based on the theory of Burton, Cabrera and Frank [7], who reduced the crystal face to a one layer interface, in which both fluid and solid cells can occur. To this two-dimensional Ising model the Onsager theory applies, predicting an order-disorder transition for the interface at an exactly solvable critical Ising temperature $\theta^{\mathrm{c}}$. This dimensionless temperature is associated with the temperature at which a crystal face roughens. In order to calculate $\theta^{\mathrm{c}}$, a procedure must be followed which is comparable to the $\mathrm{PBC}$ analysis. At first the crystal structure must be investigated, to yield the elementary growth units (ions, molecules, com- plexes) from which the crystal grows from the mother phase. Then the bonds in the first coordination sphere are determined and from these the crystal graph is defined. The crystal graph is defined as an infinite set of elements (growth units), fulfilling the symmetry of one of the 230 space groups with relations (bonds) between them. For this purpose, the growth units are reduced to centres of gravity. From the crystal graph the so-called connected nets have to be determined. A connected net in Ising language is the equivalent of an F-face in the PBC theory, and is defined as a net where all points within a slice $d_{h k l}$ are connected by uninterrupted paths of bonds. Such a connected net shows an order-disorder transition at a definite Ising temperature $\theta^{c}$, defined as:

$\theta^{\mathrm{c}}=[2 k T / \phi]^{\mathrm{c}}$,

in which $\phi$ is the bond energy of the strongest bond in the crystal graph. Below this temperature, the interface is essentially flat because a positive step free energy $\gamma_{\text {step }}$ is required to build a step. Slow growth takes place according to a layer mechanism, i.e. two-dimensional nucleation or spiral growth. Above this temperature, $\gamma_{\text {step }}$ vanishes and the interface roughens. In this case the crystal face grows fast and without barriers. The requirement of connectedness is essential for the occurrence of an order-disorder phase transition: a non-connected net, corresponding to an $\mathrm{S}$ - or K-face in PBC language, roughens at $T=0$ $\mathrm{K}$. Therefore it is always rough and will not occur as a crystal face.

Rijpkema et al. developed a formalism to derive critical Ising temperatures for complex rectangular nets [8]. In order to apply this theory, non-rectangular nets must be "rectangularized" by introducing bonds of zero and infinite strength. Once the rectangular nets are constructed and the ratio between the bond energies in the net is known, critical temperatures $\theta^{c}$ can be calculated by means of the program TC. It should be noted that the Ising model applies to nearest neighbours only, whereas in the attachment energy model all intermolecular interactions in the lattice can be taken into account. It will be investi- 
gated whether this influences the theoretical growth form. Although there is no unambiguous way to construct a crystal growth form from a series of critical Ising temperatures, it can be stated that the Ising temperature $\theta_{h k l}^{\mathrm{c}}$ increases with increasing strength of the connected net and with decreasing growth rate $R_{h k l}$. Now we assume $R_{h k l} \propto\left(\theta_{h k l}^{\mathrm{c}}\right)^{-1}$ or, if the actual $\theta$ is known, $R_{h k l} \propto\left(\theta_{h k l}^{\mathrm{c}}-\theta\right)^{-1}$ [9], which seems to be in better agreement with the experiment.

Finally, the equilibrium form of a crystal can be calculated from the relative specific surface energies $\gamma_{h k l}$ given by [10]:

$\gamma_{h k l}=C d_{h k l} \sum_{i} i E_{i}=C d_{h k l} E_{\mathrm{s}}$,

where $E_{i}$ is the interaction energy per mole of a slice $d_{l k k l}$ with the $i$ th underlying slice, $E_{\mathrm{s}}$ is the surface energy per mole and $C$ is a constant. In the case of F-faces, the terms in the series in (5) are often negligible for $i \geq 2$. Therefore, $E_{\mathrm{s}}$ can be approximated by $E_{\text {att }}$ in formula (5) because

$E_{h k l}^{\mathrm{att}}=\sum_{i} E_{i}$.

Theoretical habits are constructed by means of the program SHAPE [26] which uses centre to face distances to compute the smallest polyhedron enclosed by these faces. Theoretical growth forms are drawn by taking the central distances of the various faces directly proportional to $R_{h k l}$ according to the three different models described above:

$R_{h k l} \propto\left(d_{h k l}\right)^{-1}: \quad$ Donnay-Harker model;

$R_{h k l} \propto E_{h k l}^{\mathrm{atl}}: \quad$ attachment energy model;

$R_{h k l} \propto\left(\theta_{h k l}^{\mathrm{c}}\right)^{-1}: \quad$ Ising model.

Likewise, the equilibrium form is obtained by drawing a three-dimensional Wulff plot with central distances proportional to $\gamma_{h k l}$.

So far all bond energies between molecules were defined in reference to vacuum. Within the framework of the Hartman-Perdok theory combined with Ising models, bond energies $\phi_{i}$ of the crystal graph for crystals growing from solution are defined as:

$\phi_{i}=\phi_{i}^{\mathrm{sf}}-\frac{1}{2}\left(\phi_{i}^{\mathrm{ss}}-\phi_{i}^{\mathrm{ff}}\right)$,

where $\phi_{i}^{\mathrm{sf}}$ refers to solid-fluid bonds, $\phi_{i}^{\mathrm{ss}}$ to solid-solid and $\phi_{i}^{\text {ff }}$ to fluid-fluid bonds. The bond energies discussed thus far were the $\phi_{i}^{\text {ss }}$ bond energies. In order to equal "vacuum morphology" and "solution morphology", the socalled proportionality condition has to be introduced:

$\phi_{i} / \phi_{j}=\phi_{i}^{\mathrm{ss}} / \phi_{j}^{\mathrm{ss}}$.

In the following, our study of the morphology of $\alpha$-glycine and urea will be based on "vacuum bond energies", $\phi_{i}^{\mathrm{ss}}$. The theoretical results will be compared to real growth forms of crystals growing from solution. This is justified, taking the proportionality condition (8) into account.

\section{Concepts: intermolecular interaction}

\subsection{The charge density distribution}

In order to predict quantitatively the crystal morphology from attachment energies or critical Ising temperatures, accurate interaction energies between molecules in the crystal lattice are needed. As discussed above, the electrostatic interaction will play a major role compared with the dispersion interaction for compounds like urea and $\alpha$-glycine. Therefore, emphasis was laid on a profound description of the molecular charge density distribution $\rho(\boldsymbol{r})$, which can be obtained theoretically from a quantum-mechanical calculation of the wave function, or experimentally from $\mathrm{X}$-ray diffraction data. It is useful to decompose the molecular charge distribution into atomic charges and multipole moments, although there is by no means a unique definition. Sometimes Mulliken population analysis is used [11], a method which associates electrons with a given atom in a rather arbitrary way, depending strongly on the number of basis functions centred on the atom. For some purposes, however, Mulliken charges appear to be good enough. 
A charge partitioning method with more physical significance, defining unambiguously electric multipole tensors, was introduced by Hirshfeld [12] and is known as the stockholder recipe. In contrast to the Mulliken method, this way of partitioning is orbital-independent. It is used for both theoretical and experimental charge densities, whereas Mulliken only applies to quantumchemical calculation of the density from the wave function. In this method the molecular density at each point is partitioned among the atoms in the molecule in proportion to their respective free atom densities. For each atom a sharing function $W_{i}(\boldsymbol{r})$ is defined as:

$W_{i}(\boldsymbol{r})=\rho_{i}^{\mathrm{atom}}(\boldsymbol{r}) / \sum \rho_{j}^{\mathrm{atom}}(\boldsymbol{r})$,

where $\rho_{i}^{\text {atom }}(\boldsymbol{r})$ is the free atom density distribution of atom $i$ and $j$ runs over all the atoms in the molecule. Then, the charge density of the bonded atom $i$ is defined as:

$\rho_{i}^{\text {bonded }}(\boldsymbol{r})=W_{i}(\boldsymbol{r}) \rho^{\text {mol }}(\boldsymbol{r})$,

where $\rho^{\text {mol }}$ is the actual molecular density. The atomic deformation density $\Delta \rho_{i}(r)$ is obtained by subtracting the free atom density from the bonded atom density:

$\Delta \rho_{i}(r)=\rho_{i}^{\text {bonded }}(\boldsymbol{r})-\rho_{i}^{\text {free }}(\boldsymbol{r})$.

Net atomic charges $q_{i}$, dipole moments $\mu_{i, \alpha}$ and symmetric second moment tensors $\mu_{i, \alpha, \beta}$ are calculated from

$q_{i}=-\int \Delta \rho_{i}(r) \mathrm{d}^{3} r$,

$\mu_{i, \alpha}=-\int x_{\alpha} \Delta \rho_{i}(r) \mathrm{d}^{3} r$,

$\mu_{i, \alpha, \beta}=-\int x_{\alpha} x_{\beta} \Delta \rho_{i}(r) \mathrm{d}^{3} r$,

where $x_{\alpha}$ and $x_{\beta}$ are the $\alpha$ - and $\beta$-components of the vector $r$ measured from the atomic nucleus. The symmetric second moment tensor is related to the traceless atomic quadrupole moment tensor $\theta_{i, \alpha, \beta}$ by:

$\theta_{i, \alpha, \beta}=\frac{3}{2}\left[\mu_{i, \alpha, \beta}-\frac{1}{3} \delta_{\alpha \beta} \operatorname{Tr}\left(\mu_{i, \alpha, \beta}\right)\right]$.
All these multipole moments are symmetric with respect to permutation of suffixes. The integrals (12), (13) and (14) are evaluated numerically using Gauss quadrature.

\subsection{Electrostatic interaction}

If $R_{i, j}$ is defined as the distance between two points in the charge clouds of, respectively, atom $i$ in molecule a and atom $j$ in molecule $\mathrm{b},\left(R_{i, j}\right)^{-1}$ can be expanded as a Taylor series in $R^{-1}$, where $R$ is the distance between atoms $i$ and $j$ [13]. The electrostatic energy between molecules $a$ and $b$, represented by two sets of atomic multipoles, is then expressed as in eq. (16). In this kind of calculations it has appeared to be sufficiently accurate to employ only the first three order moments [5].

$$
\begin{aligned}
& E_{\text {clectrostatic }}^{(\mathrm{a}, \mathrm{b})} \\
& \quad=\sum_{i} \sum_{j}\left\{(R)^{-1} q_{i}^{(\mathrm{a})} q_{j}^{(\mathrm{b})}+(R)^{-3} \sum_{\alpha} R_{\alpha}\left(q_{i}^{(\mathrm{a})} \mu_{j, \alpha}^{(\mathrm{b})}\right.\right. \\
& \left.-q_{j}^{(\mathrm{b})} \mu_{i, \alpha}^{(\mathrm{a})}\right)+3(R)^{-5} \sum_{\alpha} \sum_{\beta}\left(R_{\alpha} R_{\beta}-\frac{1}{3} R^{2} \delta_{\alpha \beta}\right) \\
& \quad \times\left(\frac{1}{3} q_{i}^{(\mathrm{a})} \theta_{j, \alpha, \beta}^{(\mathrm{b})}+\frac{1}{3} q_{j}^{(\mathrm{b})} \theta_{i, \alpha, \beta}^{(\mathrm{a})}-\mu_{i, \alpha}^{(\mathrm{a})} \mu_{j, \beta}^{(\mathrm{b})}\right) \\
& -3(R)^{-7} \sum_{\alpha} \sum_{\beta} \sum_{\gamma}\left[5 R_{\alpha} R_{\beta} R_{\gamma}\right. \\
& \left.-R^{2}\left(R_{\alpha} \delta_{\beta \gamma}+R_{\beta} \delta_{\gamma \alpha}+R_{\gamma} \delta_{\alpha \beta}\right)\right] \\
& \quad \times\left(-\frac{1}{3} \mu_{i, \alpha}^{(\mathrm{a})} \theta_{j, \beta, \gamma}^{(\mathrm{b})}+\frac{1}{3} \mu_{j, \alpha}^{(\mathrm{b})} \theta_{i, \beta, \gamma}^{(\mathrm{a})}\right) \\
& +35(R)^{-9} \sum_{\alpha} \sum_{\beta} \sum_{\gamma} \sum_{\delta}\left[R_{\alpha} R_{\beta} R_{\gamma} R_{\delta}\right. \\
& +R^{4}\left(\delta_{\alpha \beta} \delta_{\gamma \delta}+\delta_{\alpha \gamma} \delta_{\beta \delta}+\delta_{\alpha \delta} \delta_{\beta \gamma}\right) \\
& -5 R^{2}\left(R_{\alpha} R_{\beta} \delta_{\gamma \delta}+R_{\alpha} R_{\gamma} \delta_{\beta \delta}+R_{\alpha} R_{\delta} \delta_{\beta \gamma}\right. \\
& \left.\left.+R_{\beta} R_{\gamma} \delta_{\alpha \delta}+R_{\beta} R_{\delta} \delta_{\alpha \gamma}+R_{\gamma} R_{\delta} \delta_{\alpha \beta}\right)\right] \\
& \left.\quad \times\left(\frac{1}{3} \theta_{i, \alpha, \beta}^{(\mathrm{a})} \theta_{j, \gamma, \delta}^{(\mathrm{b})}\right)\right\} .
\end{aligned}
$$

\subsection{Van der Waals interaction}

In order to complete the nonbonded potential function, a Van der Waals term was added to the 
Coulomb energy. The attractive dispersion energy is generally set proportional to $(R)^{-6}$, while the short-range repulsive energy can be represented in two ways: by an exponential function or a $(R)^{-n}$ function, where $n$ is often chosen equal to 9 or 12. The exponential function leads to the Buckingham potential:

$V^{\mathrm{XY}}(R)=B^{\mathrm{XY}} \exp \left(-C^{\mathrm{XY}} R\right)-A^{\mathrm{XY}} R^{-6}$.

This represents the Van der Waals interaction energy between two atoms belonging to the chemical elements $\mathrm{X}$ and $\mathrm{Y}$, separated by a distance $R, A^{\mathrm{XY}}, B^{\mathrm{XY}}$ and $C^{\mathrm{XY}}$ are the nonbonded parameters, subject to the known combining laws. This potential was used in one model for $\alpha$ glycine. The $R^{-n}$ model leads to the LennardJones $6-n$ potential:

$V^{\mathrm{XY}}(R)=-A^{\mathrm{XY}} R^{-6}+B^{\mathrm{XY}} R^{-n}$.

For urea and two other models of $\alpha$-glycine a Lennard-Jones 6-9 potential was chosen, with parameters from Hagler, Huler and Lifson [14] derived for amide crystals.

\section{Application to $\alpha$-glycine}

\subsection{Structure and intermolecular interactions}

$\alpha$-Glycine crystallizes in the form of the zwitterion $\mathrm{NH}_{3}^{+} \mathrm{CH}_{2} \mathrm{COO}^{-}$in the monoclinic space group $\mathrm{P} 2{ }_{1} / \mathrm{n}(a=5.084 \AA, b=11.820 \AA, c=$ $\left.5.458 \AA, \beta=111.95^{\circ}\right)[15]$. The unit cell contains four molecules centred at:

$\mathrm{M}(1) \quad x, y, z$,

$\mathrm{M}(2) \frac{1}{2}+x, \frac{1}{2}-y, \frac{1}{2}+z$,

$\mathrm{M}(3)-x,-y,-z$,

M(4) $\frac{1}{2}-x, \frac{1}{2}+y, \frac{1}{2}-z$,

with $x \simeq 0.121, y \simeq 0.118$ and $z \simeq-0.018$. The crystal structure has been drawn in fig. 1 . The extinction conditions for the space group concerned are given by [16]:

$h 0 l: h+l=2 n ; \quad h 00: h=2 n ; \quad 0 k 0: k=2 n ;$

$00 l: l=2 n$.

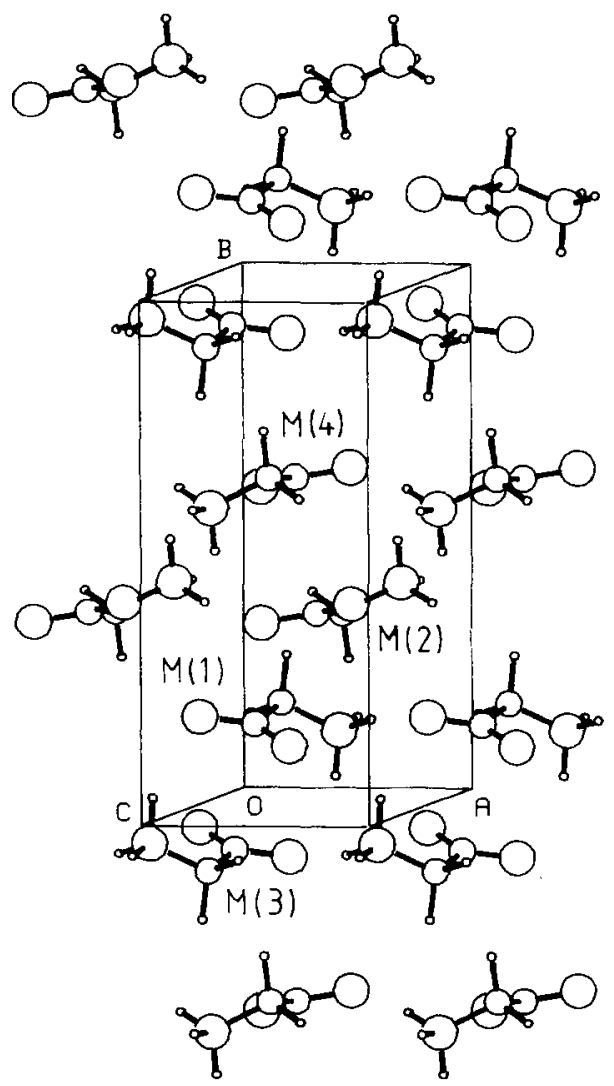

Fig. 1. Packing arrangement of $\alpha$-glycine.

Considering short intermolecular bond distances, six different types of nearest neighbours can be distinguished in the first coordination sphere of $\mathrm{M}(1)$. These are listed in table 1 and drawn in the crystal graph in fig. 2 . In order to

Table 1

Nearest neighbours of $\mathrm{M}(1)$ in $\alpha$-glycine; the numbering of the molecules refers to fig. 2

\begin{tabular}{llll}
$\begin{array}{l}\text { Bond } \\
\text { type }\end{array}$ & Bond & Contact & $\begin{array}{l}\text { Short distance } \\
(\AA)\end{array}$ \\
\hline a & $\mathrm{M}(1)-\mathrm{M}(3)$ & $\mathrm{N}-\mathrm{H} \cdots$ O bond & 3.020 \\
b & $\mathrm{M}(1)-\mathrm{M}(1,001)$ & $\mathrm{N}-\mathrm{H} \cdots$ O bond & 2.767 \\
& $\mathrm{M}(1)-\mathrm{M}(1,00 \overline{1})$ & & \\
c & $\mathrm{M}(1)-\mathrm{M}(3,100)$ & $\mathrm{N}-\mathrm{H} \cdots$ O bond & 2.936 \\
d & $\mathrm{M}(1)-\mathrm{M}(2,00 \overline{1})$ & $\mathrm{C}-\mathrm{H} \cdots$ O bond & 3.295 \\
& $\mathrm{M}(1)-\mathrm{M}(2,100)$ & & \\
e & $\mathrm{M}(1)-\mathrm{M}(1,100)$ & $\mathrm{N}-\mathrm{H} \cdots$ O bond & 2.833 \\
& $\mathrm{M}(1)-\mathrm{M}(1, \overline{1} 00)$ & & \\
f & $\mathrm{M}(1)-\mathrm{M}(2)$ & $\mathrm{O}-\mathrm{O}$ contact & 3.680
\end{tabular}




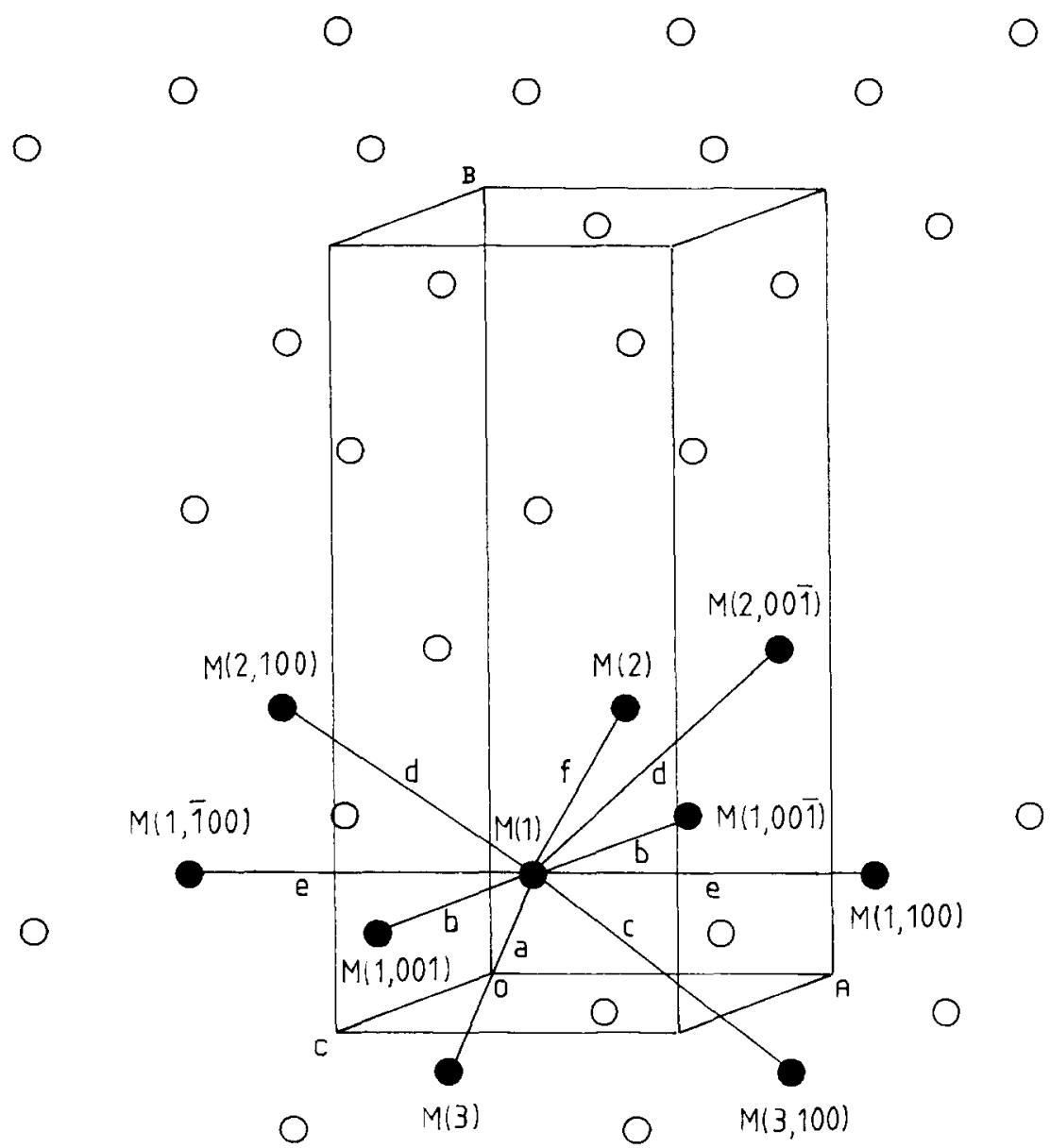

Fig. 2. Crystal graph of the $\alpha$-glycine structure with nearest neighbour bonds of M(1). Molecules are represented by their centres of mass.

calculate intermolecular interactions, three different atom-atom potential parameter sets were used:

In model 1 atomic Mulliken charges $\left(q_{i}\right)$ were used which were obtained by Voogd et al. [17] from Hartree-Fock calculations with a DZP basis set. These were combined with nonbonded parameters for a Buckingham potential which were calculated by fitting model parameters to the lattice energy and keeping the above-mentioned charges fixed [18].

In model 2 a potential energy function for $\alpha$-glycine defined by Berkovitch-Yellin [5] was used: the electrostatic term was represented by a multipole expansion (up to the quadrupole mo- ment) of an experimental charge density distribution derived from low temperature X-ray diffraction data. The Van der Waals term was given by a Lennard-Jones (6-9) potential with parameters from ref. [14]. The intermolecular energies in ref. [5] could be reproduced by our calculations, although a mistake was found in ref. [5] concerning the picture of the local coordinate systems (in which the multipole moments are defined) on atoms $\mathrm{C} 2$ and $\mathrm{H} 1$. Therefore it is presented here correctly in fig. 3 .

For model 3 a theoretical charge distribution was derived from ab initio Hartree-Fock (GAMESS [24]) calculations with a $6-31 \mathrm{G}^{* *}$ basis set. The difference density map (drawn in the 

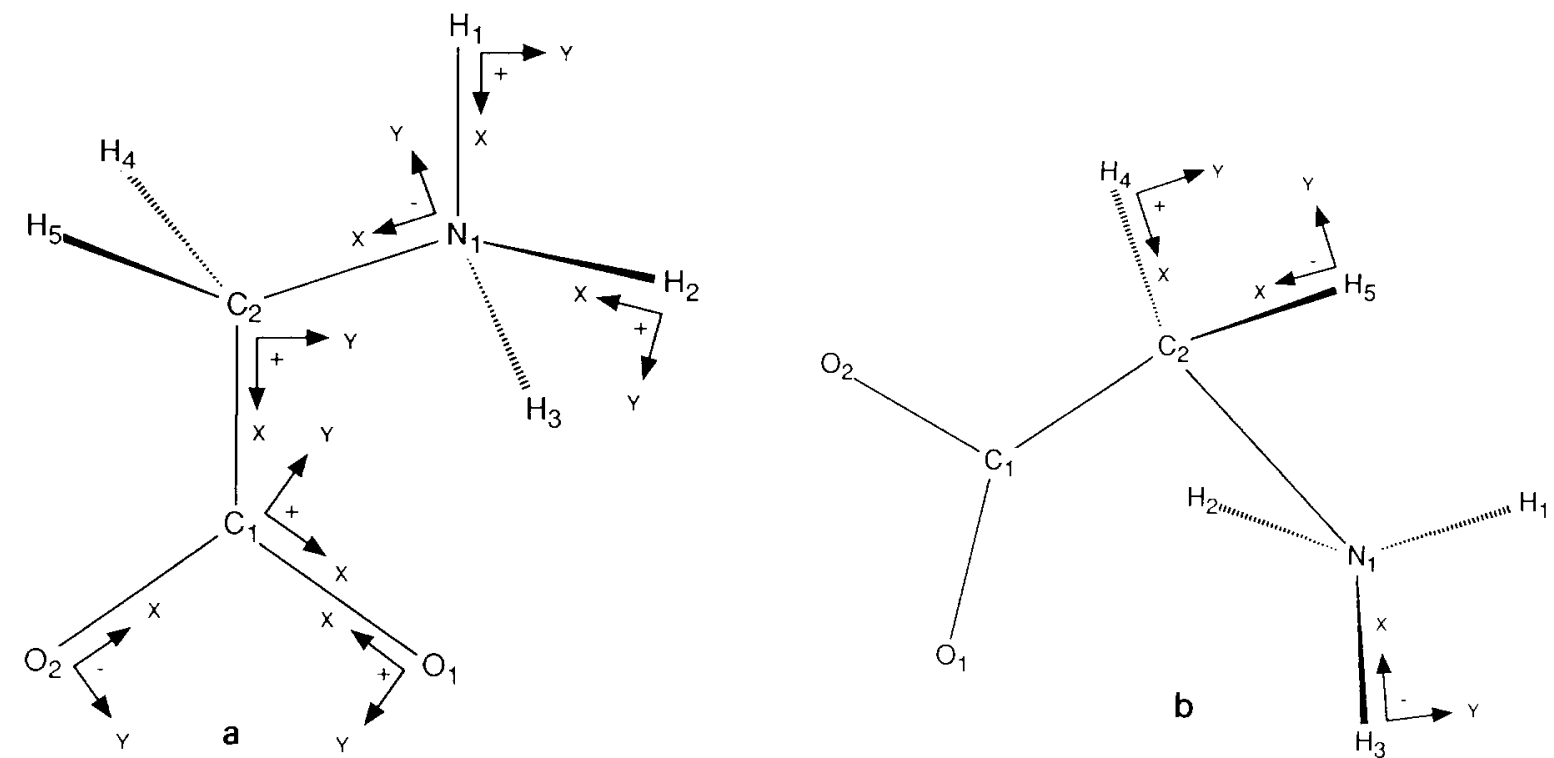

Fig. 3. Local coordinate systems $x, y, z$ centred on the atoms of $\alpha$-glycine, as used in model 2, corrected with respect to ref. [5]. A + or - sign denotes a right- or left-handed coordinate system, respectively.

plane defined by $\mathrm{C} 1, \mathrm{C} 2$ and $\mathrm{N}$ ) is shown in fig. 4 . The charge density was expanded into multipoles according to the stockholder method (table 2). The associated Van der Waals parameter set was chosen to be the same as in model 2.

Comparing models 1,2 and 3 , it appears that the atomic point charges are quite different. Consequently, there are differences between the corresponding intermolecular energies (table 3 ), as the main contribution is formed by the monopole-monopole interactions. The relative values per model, however, are comparable. It will be clear that bond a suggests a strong dimer association, while bond $\mathrm{f}$ is a repulsive and cannot be considered as a "strong bond" in the following PBC analysis.

\subsection{Qualitative $P B C$ analysis}

From the "strong bonds" defined in table 1, seven PBCs in five different directions were obtained (table 4). In a structure projection along [001] (fig. 5) several F-slices can be found by combining PBC [001] with other ones. If the $\mathrm{M}(1)-\mathrm{M}(3)$ dimer is taken as elementary growth unit, PBCs [001] are bonded to neighbouring
PBCs in the slices $(020)$ and $(110)$ by means of PBCs $[100] \alpha$ and [1111], respectively. If this association is abandoned, also $(020)_{\mathrm{b}}$ (differing $\frac{1}{2} d_{020}$ from slice $(020)_{i a}$ in level) and (120) are F-slices by means of PBCs in [100] $\beta$ and [211] directions, respectively.

By means of the computer program BEHNG2 [25], structure projections on several faces have been made, which are shown in fig. 6. The molecules are represented by dots, which are connected to nearest neighbours within the slice by means of different types of lines. If it is possible to travel via these bonds from every molecule in the slice to any other, the slice is an F-slice or connected net. From these projections it can be seen that (110) and (120) are indeed connected nets, while for (020) two different Fslice configurations as possible, Furthermore, it appears that (011) and (101) are also F-slices, while (200) and (002) are S-faces and (101) is a K-face.

\subsection{Quantification of the crystal morphology}

\subsubsection{Attachment energy model}

Using the computer program MOLINT, attachment and slice energies have been deter- 


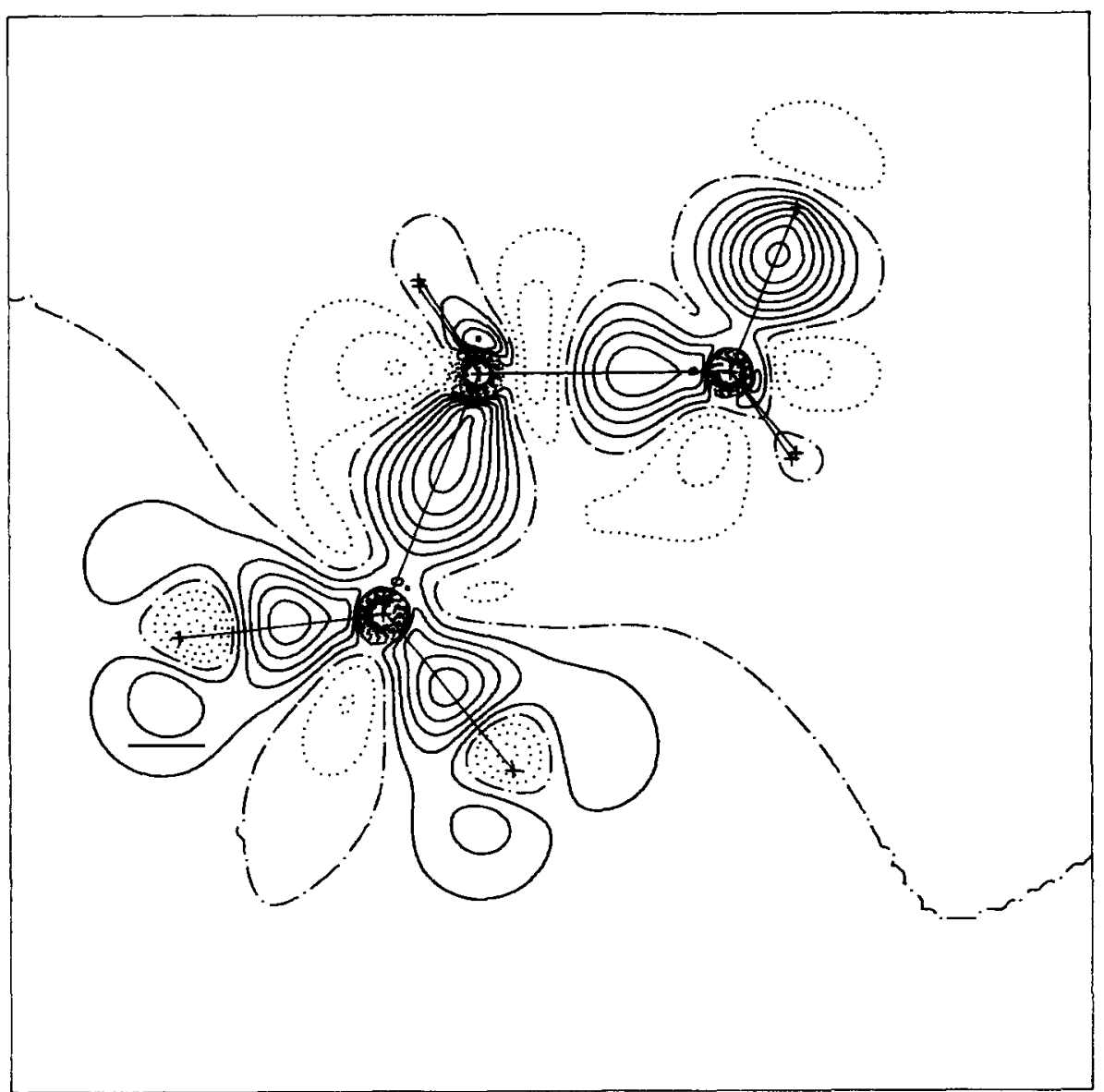

Fig. 4. Deformation (difference) density map of the $\alpha$-glycine zwitterion in the plane of $N$ (above right), $C_{2}$ (middle) and $C_{1}$ (below left). The contour interval amounts to $0.10 \mathrm{e} \AA^{-3}$. (—) Positive contours (electron excess); $\left(\cdot \cdot_{-} \cdot \cdot\right)$ zero contours; $(\cdots \cdots)$ negative contours (electron deficiency).

Table 2

Electrostatic properties of $\alpha$-glycine $\left(\times 10^{4}\right)$; net atomic charges $q(e)$, dipole moments $d(e \AA)$ and quadrupole moments $\mu\left(e \AA^{2}\right)$ are defined in a Cartesian molecular axial system with $x$ along $N \rightarrow \mathrm{C} 2$, and $z$ along the normal to the molecular plane given by the vector product $(\mathrm{C} 2 \rightarrow \mathrm{C} 1) \times(\mathrm{C} 2 \rightarrow \mathrm{N})$

\begin{tabular}{|c|c|c|c|c|c|c|c|c|c|c|}
\hline Atom & $q$ & $d_{x}$ & $d_{y}$ & $d_{z}$ & $\mu_{x x}$ & $\mu_{y y}$ & $\mu_{z z}$ & $\mu_{x y}$ & $\mu_{y z}$ & $\mu_{x z}$ \\
\hline$\overline{\mathrm{C} 1}$ & 1845 & -657 & 34 & -20 & -753 & -751 & 1505 & 222 & -118 & 913 \\
\hline $\mathrm{C} 2$ & 394 & 29 & 391 & -149 & 506 & -282 & -225 & 296 & -68 & -55 \\
\hline $\mathrm{H} 1$ & 1796 & -1002 & 45 & -158 & -31 & -59 & 90 & -51 & 15 & 23 \\
\hline $\mathrm{H}_{2}$ & 1893 & 133 & 848 & 572 & 25 & -126 & 101 & -102 & -30 & 49 \\
\hline $\mathrm{H} 3$ & 1879 & 385 & 140 & -971 & 22 & -37 & 15 & -48 & 41 & -37 \\
\hline $\mathrm{H} 4$ & 560 & -247 & -116 & 705 & 83 & 1 & -85 & 2 & 32 & -64 \\
\hline $\mathrm{H} 5$ & 531 & -214 & -587 & -407 & 58 & -35 & -23 & -24 & -22 & 57 \\
\hline N1 & 702 & 38 & -33 & 20 & -230 & -25 & 255 & -43 & 1 & 57 \\
\hline O1 & -4903 & -574 & -1120 & 59 & -66 & 199 & -132 & -309 & 122 & -8 \\
\hline $\mathrm{O} 2$ & -4698 & -384 & 1095 & -127 & 315 & -323 & 8 & 187 & -73 & -136 \\
\hline
\end{tabular}


Table 3

Intermolecular energies for $\alpha$-glycine in different models; the number of the molecules refers to fig. 2

\begin{tabular}{llrrr}
\hline $\begin{array}{l}\text { Bond } \\
\text { type }\end{array}$ & Neighbour & \multicolumn{3}{c}{ Intermolecular energy $(\mathrm{kJ} / \mathrm{mol})$} \\
\cline { 2 - 5 } & & \multicolumn{1}{c}{ Model 1 } & Model 2 & Model 3 \\
\hline a & M(1)-M(3) & -146.67 & -55.10 & -126.99 \\
b & M(1)-M(1,001) & -94.00 & -31.78 & -88.12 \\
c & M(1)-M(3,100) & -136.46 & -29.39 & -97.53 \\
d & M(1)-M(2,00) & -30.19 & -13.44 & -23.02 \\
e & M(1)-M(1,100) & -10.46 & -2.37 & -0.44 \\
f & M(1)-M(2) & +32.62 & +15.70 & +54.40 \\
\hline
\end{tabular}

mined for all possible F-slices in the three different charge models described above. By summing intermolecular energies over 6 cells in the $|x|$ direction, 3 cells in the $|y|$-direction and 6 cells in the $|z|$-direction, convergence of the lattice sum is assured within a summation limit of $30 \AA$. The attachment energies for the three models are listed in table 5, from which slice energies can easily be derived by means of eq. (1). For the F-faces, the results for model 2 agree with those obtained by Berkovitch-Yellin [5]. Small differences are due to a different summation limit. Also, calculations for the (200), (002) and (101) slices were performed, but these results are in conflict with ref. [5]. Attachment energies calculated for these faces are $10-30 \mathrm{~kJ} / \mathrm{mol}$ more negative than reported in ref. [5] (table 5). As shown in fig. 6 , these three slices do not have F-character. The numerical differences, however, cannot be explained by this qualitative consideration but perhaps by differently chosen slice boundaries. According to the classical HartmanPerdok PBC theory, these faces do not appear on the theoretical growth form anyway.

In model 1 it was assumed that in the gas phase glycine molecules are present as non-zwitterions, while in the solid state they are zwitterions. So, during crystallization a proton has to be transferred from the $\mathrm{COOH}$ group to the $\mathrm{NH}_{2}$ group. Therefore, a proton-transfer energy term $U_{\mathrm{pr}}$, defined as the energy difference between the zwitterion and the non-zwitterion, was taken into account in the calculation of the crystal energy $E_{\mathrm{cr}}$ and the atom-atom potentials [18]:

$E_{\mathrm{cr}}=-H_{\mathrm{sub}}+U_{\mathrm{pr}}-2 R T$,

where $H_{\text {sub }}$ is the experimental enthalpy of sublimation $(145 \mathrm{~kJ} / \mathrm{mol})$ and the calculated SCF energy $U_{\mathrm{pr}}$ amounts to $-156 \mathrm{~kJ} / \mathrm{mol}$ [17]. The term $2 R T$ represents a correction for the difference between the gas phase enthalpy $(p V+3 R T)$ and the vibrational contribution to the crystal enthalpy $(6 R T)$ [14]. This results in a value of $-306 \mathrm{~kJ} / \mathrm{mol}$ for $E_{\mathrm{cr}}$.

Table 4

Schematic configuration of the PBCs in $\alpha$-glycine

\begin{tabular}{lll}
\hline PBC & Constituting molecules & Bonds \\
\hline$[001]$ & $\mathrm{M}(1)-\mathrm{M}(1,001)$ & $\mathrm{b}$ \\
{$[100] \alpha$} & $\mathrm{M}(1, \overline{1} 00)-\mathrm{M}(1)$ & $2 \mathrm{a}+\mathrm{c}+2 \mathrm{e}$ \\
& $\mathrm{M}(3, \overline{1} 00)-\mathrm{M}(3)$ & \\
{$[100] \beta$} & $\mathrm{M}(2, \overline{1} 00)-\mathrm{M}(2)$ & $2 \mathrm{~d}+2 \mathrm{e}$ \\
& $\mathrm{M}(1)-\mathrm{M}(1,100)$ & $2 \mathrm{~d}$ \\
{$[10 \overline{1}]$} & $\mathrm{M}(1)-\mathrm{M}(2, \overline{1} 00)-\mathrm{M}(1, \overline{1} 01)$ & $2 \mathrm{a}+2 \mathrm{~d}$ \\
{$[11 \overline{1}]$} & $\mathrm{M}(1)-\mathrm{M}(2,00 \overline{1})-\mathrm{M}(4,00 \overline{1})-\mathrm{M}(3,11 \overline{1})-\mathrm{M}(1,11 \overline{1})$ & $2 \mathrm{a}+2 \mathrm{~d}$ \\
{$[\overline{1} 11]$} & $\mathrm{M}(1)-\mathrm{M}(2, \overline{1} 00)-\mathrm{M}(4, \overline{1} 00)-\mathrm{M}(3, \overline{1} 11)-\mathrm{M}(1, \overline{1} 11)$ & $\mathrm{a}+\mathrm{c}+2 \mathrm{~d}$ \\
{$[\overline{2} 11]$} & $\mathrm{M}(1)-\mathrm{M}(2, \overline{1} 00)-\mathrm{M}(4, \overline{1} 00)-\mathrm{M}(3, \overline{1} 11)-\mathrm{M}(1, \overline{2} 11)$ &
\end{tabular}




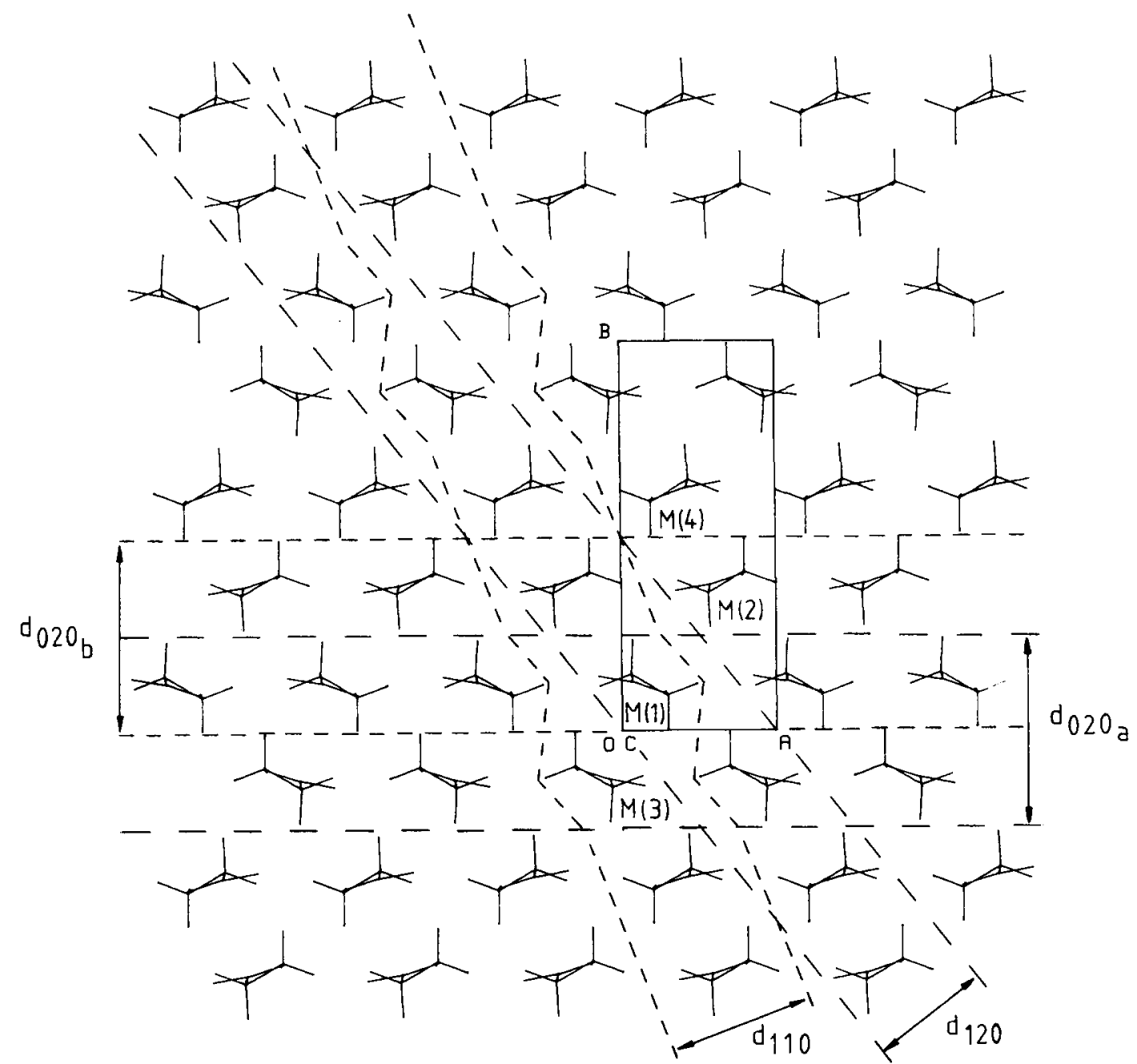

Fig. 5. Projection of the $\alpha$-glycine structure along the $c$-axis. Only the molecular bond frame is drawn. PBCs are bonded in the slices $d_{020 \mathrm{a}}, d_{020 \mathrm{~b}}, d_{110}$ and $d_{120}$ so these are F-slices.

In models 2 and 3 the proton-transfer energy is not taken explicitly into account. In these cases $E_{\text {cr }}$ can be related to $H_{\text {sub }}$ by:

$E_{\mathrm{cr}}=-H_{\mathrm{sub}}-2 R T$.

In order to compare the three models it is therefore necessary to subtract the term $U_{\mathrm{pr}}$ from the total crystal energy $E_{\text {cr }}$ in model 1 . This results in an "experimental" crystal energy of -150 $\mathrm{kJ} / \mathrm{mol}$, which compares reasonably to the calculated values for $E_{\mathrm{cr}}$ in models 2 and 3, -111 and $-226 \mathrm{~kJ} / \mathrm{mol}$, respectively.
A problem arises when the proton-transfer energy term has to be distributed among attachment and slice energy. We assume that in the crystal growth process, the molecules in the adsorption layer already have the zwitterion structure. Therefore the attachment energies in table 5 remain unchanged for model 1 . In fact, however, growth occurs in the kinks. In this case it is difficult, or perhaps even impossible, to calculate the distribution of $U_{\mathrm{pr}}$ among slice and attachment energy.

Although the attachment energies of the vari- 


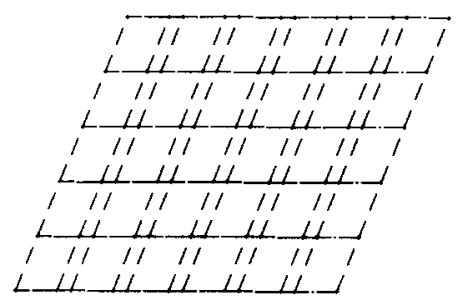

a

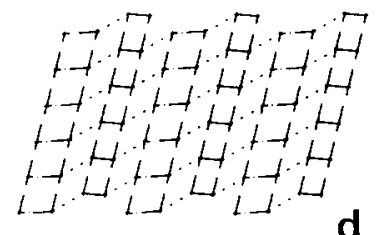

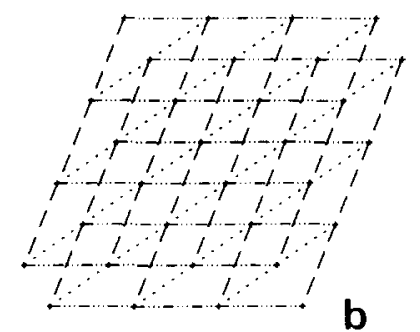

b
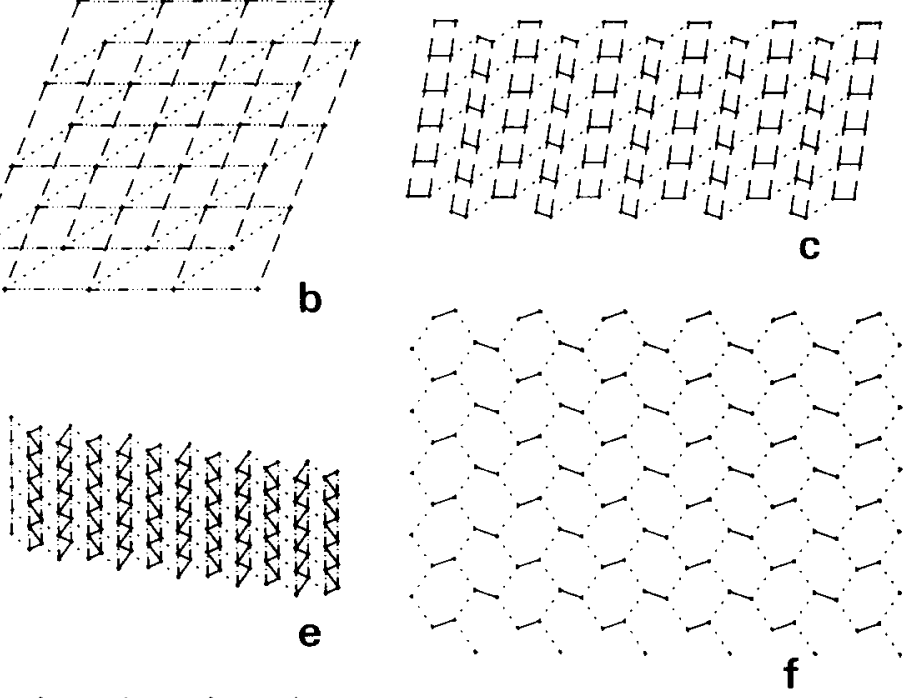

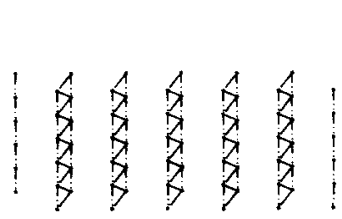

g

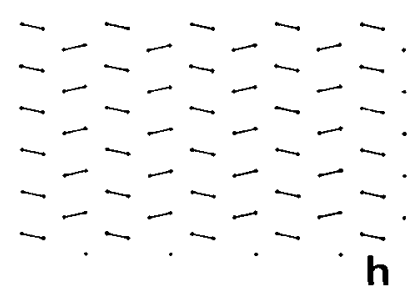

$H$

$\begin{array}{ll}H & H \\ H & H \\ H & H \\ H & H\end{array}$

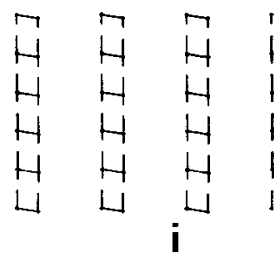

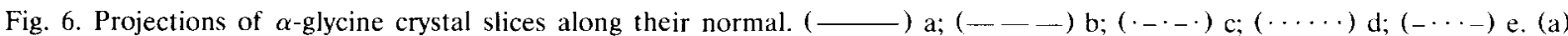
Connected net of $(020)_{a}$, rectangularized in fig. $7 \mathrm{a} ;(\mathrm{b})$ connected net of $(020)_{b}$, rectangularized in fig. $7 \mathrm{c}$; (c) rectangular connected net of (110); (d) rectangular connected net of (120); (e) connected net of $(011)$, rectangularized in fig. $7 \mathrm{~d}$; (f) triangular connected net of (101), rectangularized in fig. $7 \mathrm{~b}$; (g) S-face configuration of (200); (h) K-face configuration of (101); (i) S-face configuration of (002).

Table 5

Attachment energies $E_{h k l}^{\text {att }}$ for $\alpha$-glycine for nearest neighbour bonds and in the summation limit of $30 \AA$

\begin{tabular}{|c|c|c|c|c|}
\hline \multirow[t]{2}{*}{ Face } & \multirow{2}{*}{$\begin{array}{l}\text { Nearest neighbour } \\
\text { bonds per molecule }\end{array}$} & \multicolumn{3}{|c|}{$E_{h k l}^{\mathrm{att}}(\mathrm{kJ} / \mathrm{mol})$} \\
\hline & & Model 1 & Model 2 & Model 3 \\
\hline$(020)_{\mathrm{a}}$ & $\mathrm{d}$ & -56.89 & -27.28 & -30.09 \\
\hline$(020)_{b}$ & $(a+c) / 2$ & -113.88 & -40.44 & -76.20 \\
\hline$(110)$ & $(2 c+2 d+4 e) / 4$ & -114.06 & -42.25 & -67.41 \\
\hline$(120)$ & $(a+c+2 d+4 e) / 4$ & -126.00 & -50.06 & -83.36 \\
\hline$(011)$ & $(4 b+2 d) / 4$ & -126.84 & -55.10 & -102.10 \\
\hline$(101)$ & $(4 b+2 c+2 e) / 4$ & -185.26 & -60.53 & -122.32 \\
\hline$(200)$ & & - & -54.16 & - \\
\hline$(002)$ & & - & -63.97 & - \\
\hline$(10 \overline{1})$ & & - & -78.44 & - \\
\hline$E_{\mathrm{cr}}($ Coulomb $)$ & & -205.03 & -63.09 & -173.25 \\
\hline$E_{\mathrm{cr}}(\mathrm{vdWaals})$ & & -100.51 & -47.43 & -52.55 \\
\hline$E_{\mathrm{cr}}($ total $)$ & $(a+2 b+c+2 d+2 e) / 2$ & -305.54 & -110.52 & -225.80 \\
\hline
\end{tabular}


ous faces are quite different for the three models, the relative magnitudes, and therefore the relative theoretical growth rates, are similar. Thus the theoretical growth forms are quite comparable: all models show the pinacoid $(020)$ and the prisms (110) and (011). For the (020) face, where two different slice configurations are possible, it was assumed that growth is controlled by the most stable layer $(020)_{a}$. The growth form based on model 1 (which should be reliable because the atom-atom potentials were obtained by a fit to experimental data) is shown in fig. 8a. When the growth form is constructed according to ref. [5], including the non-F-faces (200), (002) and (101) with our attachment energies, a different habit is obtained compared to the growth form reported

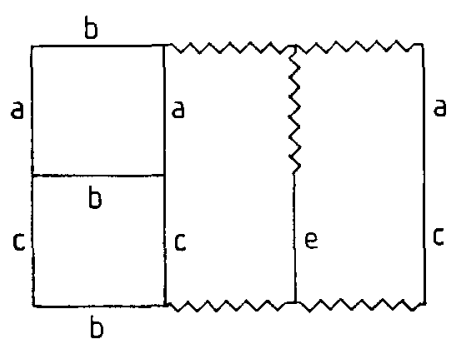

a

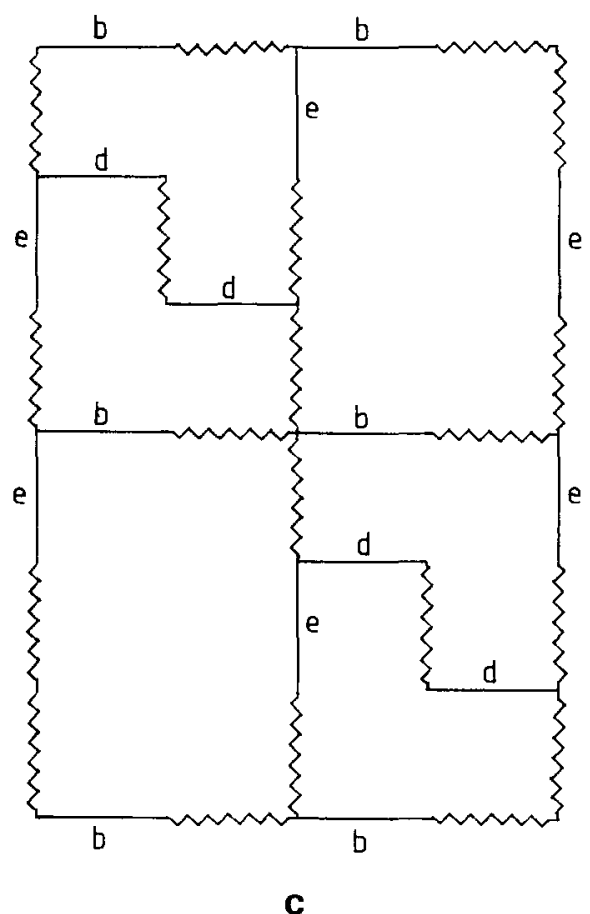

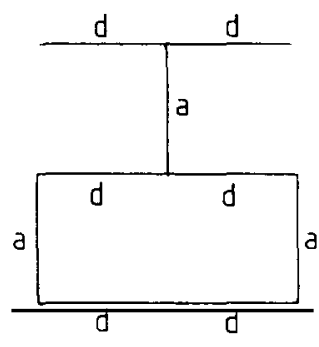

b

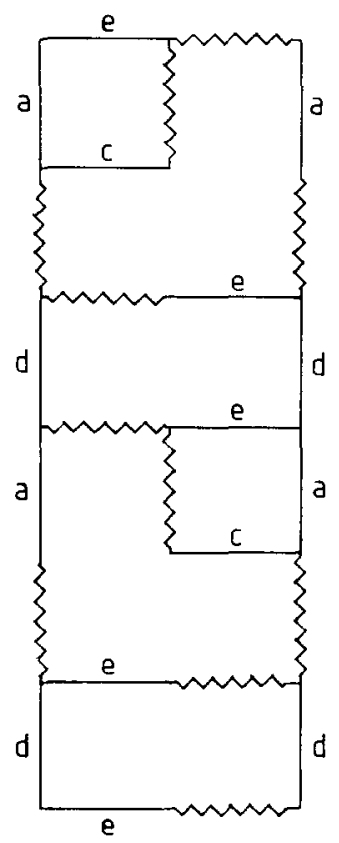

d

Fig. 7. Rectangularization of planar connected nets. By introducing bonds of infinite strength (wiggled bonds in the figures) nodes are cut at appropriate points. (a) Rectangularized connected net of $(020)_{a}$; (b) rectangularized connected net of (101); (c) rectangularized connected net of $(020)_{b}$; (d) rectangularized connected net of $(011)$. 

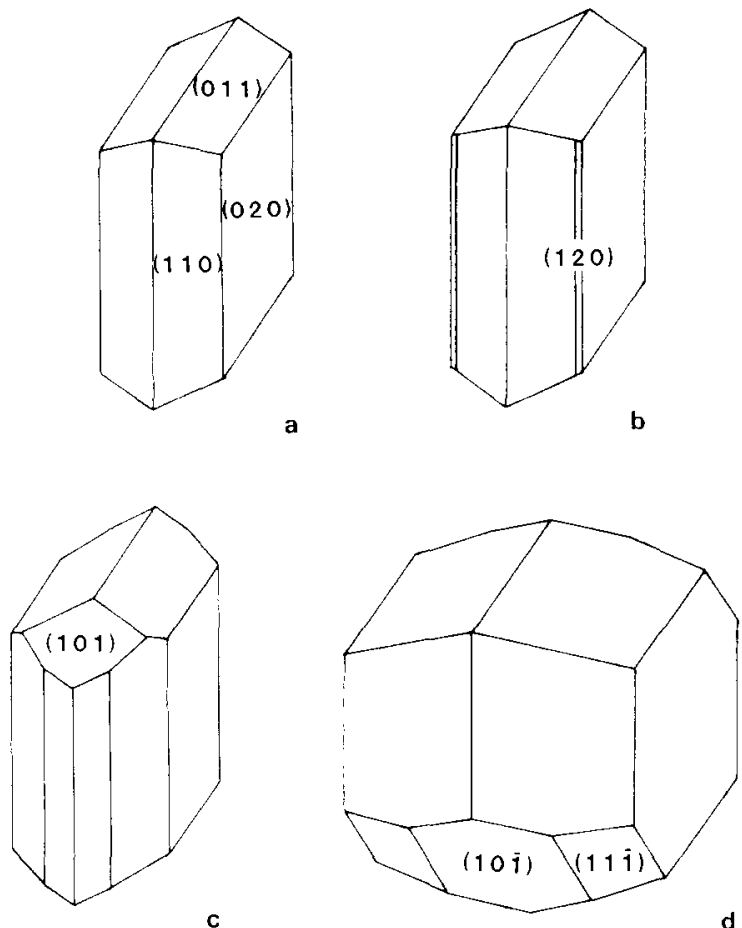

Fig. 8. Theoretical habits of $\alpha$-glycine: (a) growth form according to the $E_{\text {att }}$ model; (b) growth form according to the Ising model; (c) equilibrium form; (d) growth form according to the Donnay-Harker model.

in ref. [5]: the large (001) face in ref. [5] has totally disappeared, while the $(10 \overline{1})$ face has become much smaller. Thus it resembles the growth forms obtained from a structural PBC analysis, as shown in fig. 8a. Also growth forms based on $E_{\text {att }}$ taking only nearest neighbour bonds into account (table 5) show this shape.

\subsubsection{Ising model}

Critical Ising temperatures $\theta_{h k l}^{c}$ have been calculated using the computer program $\mathrm{TC}$ written by Rijpkema et al. [8], which is based on nearest neighbour interactions only. The relative bond strengths needed for this purpose were taken from table 2. As the roughening of an existing, i.e. protonated, layer is considered and only interaction between nearest neighbours plays a role, $U_{\mathrm{pr}}$ is not involved in model 1.

Rectangularization of the nets is shown in fig. 7. The critical temperatures calculated for the
Table 6

Critical Ising temperatures $\theta_{h k l}^{c}$ for charge models 1,2 and 3

\begin{tabular}{|c|c|c|c|}
\hline \multirow[t]{2}{*}{$\overline{N e t}$} & \multicolumn{3}{|l|}{$\theta_{h k !}^{c}$} \\
\hline & Model 1 & Model 2 & Model 3 \\
\hline$(020)_{a}$ & 2.1142 & 1.6920 & 1.9960 \\
\hline$(020)_{\mathrm{h}}^{\mathrm{a}}$ & 0.6748 & 0.6013 & 0.5071 \\
\hline$(110)$ & 1.1947 & 1.1779 & 1.2092 \\
\hline$(120)$ & 1.1862 & 1.1038 & 1.1820 \\
\hline$(011)$ & 0.9709 & 0.8104 & 0.7937 \\
\hline$(101)$ & 0.4581 & 0.5333 & 0.4060 \\
\hline
\end{tabular}

three different charge models are presented in table 6 . The theoretical growth forms based on the assumption $R_{h k l} \propto\left(\theta_{h k l}^{\mathrm{c}}\right)^{-1}$ appear to be very much alike for the three different models again. The habit based on model 1 is drawn in fig. 8b.

\subsubsection{Equilibrium form}

The equilibrium form based on $R_{h k l} \propto \gamma_{h k l}$, taking the attachment energy per surface unit $E_{h k l}^{\text {att }} \cdot d_{h k l}$ as a measure for the relative surface energy $\gamma_{h k l}$ (table 7), is drawn in fig. 8c.

\subsubsection{Donnay-Harker model}

The growth morphology based on the Donnay-Harker law is constructed in fig. 8d, from slice thickness data as listed in table 7 .

\subsection{Comparison of theoretical and experimental morphologies}

$\alpha$-Glycine crystals grown from aqueous solution are bipyramidal with $(020),(110),(120)$ and

Table 7

Connectedness (c) or non-connectedness (nc) of $\alpha$-glycine nets, $1 / d_{h k l}$ values and relative specific surface energies $\gamma_{h k l}$ (model 1)

\begin{tabular}{llll}
\hline Face & Connectedness & $\begin{array}{l}1 / d_{h k l} \\
\left(\AA^{-1}\right)\end{array}$ & $\gamma_{h k l}$ \\
\hline$(020)$ & $\mathrm{c}$ & 0.169 & 1.000 \\
$(011)$ & $\mathrm{c}$ & 0.215 & 1.754 \\
$(110)$ & $\mathrm{c}$ & 0.228 & 1.486 \\
$(10 \overline{1})$ & $\mathrm{nc}$ & 0.229 & - \\
$(1 \overline{1} 0)$ & $\mathrm{nc}$ & 0.244 & - \\
$(120)$ & $\mathrm{c}$ & 0.271 & 1.383 \\
$(101)$ & $\mathrm{c}$ & 0.339 & 1.625 \\
\hline
\end{tabular}



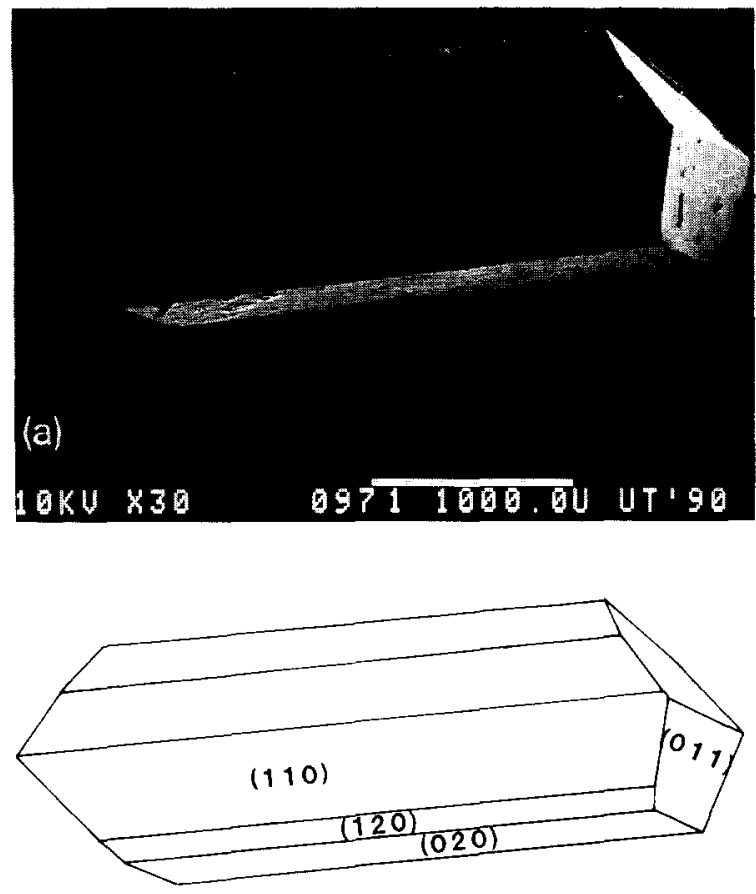

(b)

Fig. 9. (a) $\alpha$-Glycine crystal grown from aqueous solution; (b) The crystal indexed on an Enraf-Nonius CAD4 diffractometer.

(011) as most important faces (fig. 9). This is in good agreement with theoretical growth forms derived from the Ising and attachment energy models as well as with the equilibrium form. The Donnay-Harker model shows two faces which are not found on the real crystal. The (120) face is well developed on the crystals from aqueous solution, while on the theoretical form it is very small; this is ascribed to adsorption of water on the relatively polar face, thus inhibiting the growth.

Glycine crystals grown by sublimation, however, seem to show (001) and (101) as top faces according to experiments reported by BerkovitchYellin [5]. These are not F-faces, so according to the PBC theory they do not occur on the theoretical growth form based on Ising or $E_{\text {att }}$ models. Attachment energies calculated for these faces are much more negative than reported in ref. [5]. When these non-F-faces are included in the construction of a growth form they appear indeed to be absent, on account of their high attachment energies. Therefore it is concluded that external factors like the presence of impurities might have given rise to these pseudo-F-faces reported in ref. [5].

Finally, it appears that there is little variation between Ising and $E_{\text {att }}$ growth forms based on quite different charge distribution models.

\section{Application to urea}

\subsection{Introduction}

Urea crystallizes in the tetragonal space group $\mathrm{P} \overline{4} 2{ }_{1} \mathrm{~m}(a=5.661 \AA, c=4.712 \AA)[19]$. The unit cell contains two molecules centred at:

$$
\begin{array}{ll}
\mathrm{M}(1) & \left(0, \frac{1}{2}, z\right), \\
\mathrm{M}(2) & \left(\frac{1}{2}, 0,1-z\right) \text { with } \quad z \approx 0.32 .
\end{array}
$$

The extinction conditions for the space group in question are given by:

$h 00: h=2 n ; \quad h k 0: h+k=2 n$.

\subsection{Charge densities and intermolecular interac- tions}

For the urea monomer, a Hartree-Fock (GAMESS [25]) calculation was performed with a $6-31 \mathrm{G}^{* *}$ basis set. The molecular dipole moment was calculated to be $4.97 \mathrm{D}$, directed from $\mathrm{O}$ to C. For urea in the crystal, a somewhat greater experimental value of 5.4 D was found [20], probably due to hydrogen bonding in the crystal structure. The deformation density map of the urea molecule, featuring the charge density of the molecule minus the density of the free atoms, is shown in fig. 10. The molecular electron density was partitioned into net atomic charges, dipole and quadrupole moments according to the stockholder method (table 8). Higher order moments play a minor role. Electrostatic interaction energies between reference molecule $\mathrm{M}(1)$ and a number of neighbours in the crystal are given in table 9. When these results are compared to calculations of Spackman et al. [20], based on 


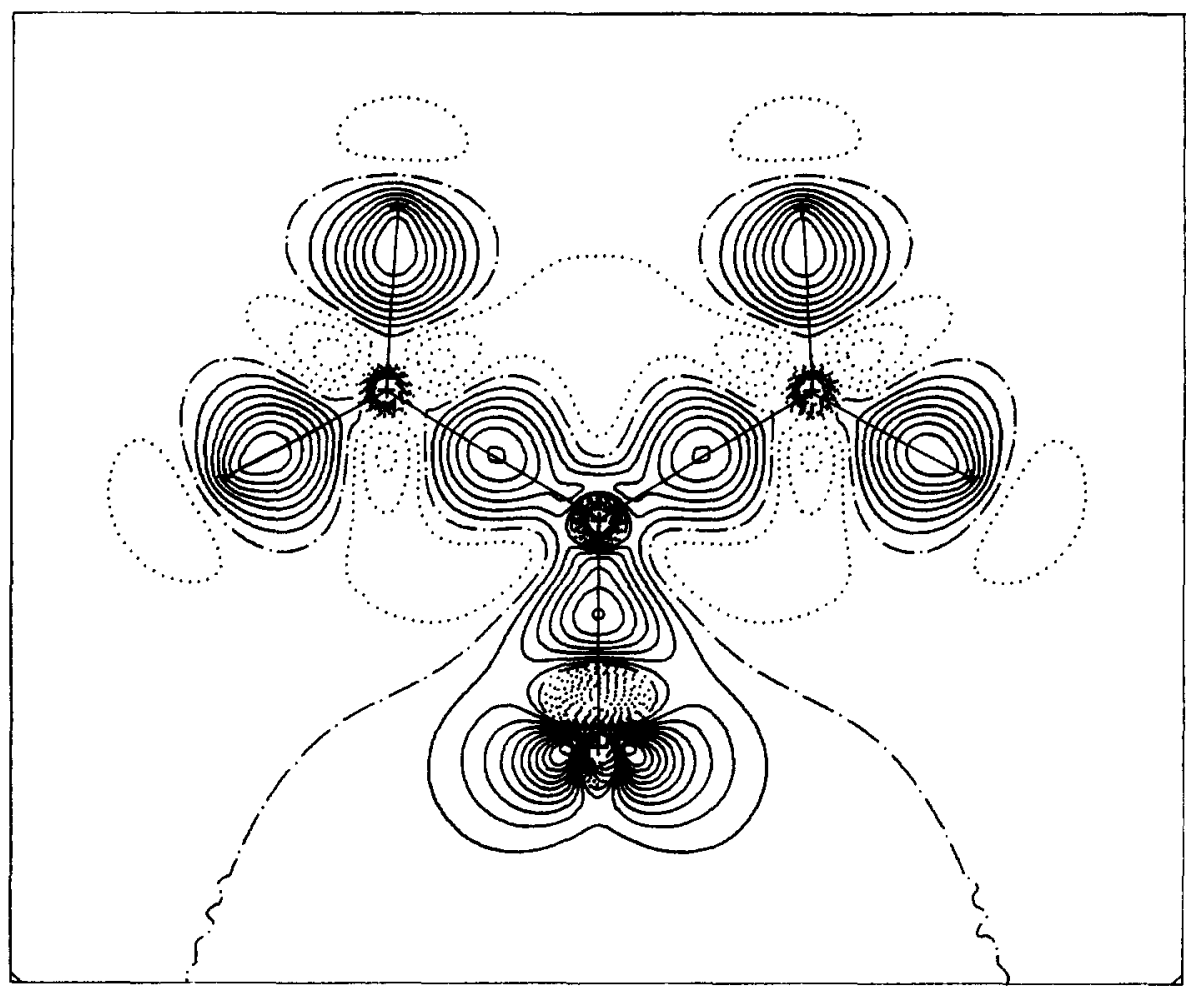

Fig. 10. Deformation (difference) density map of the urea molecule in the plane of the molecule. The contour interval amounts to 0.10 e $\AA^{-3} .(-)$ Positive contours (electron excess); $(\cdot-\cdot \cdot)$ zero contours; $(\cdots \cdots)$ negative contours (electron deficiency).

X-ray and neutron diffraction data, it appears that the agreement between theoretical and experimental values is better than might be expected regarding the large experimental estimated standard deviations (table 10). The Van der Waals interaction energy was calculated by means of a Lennard-Jones (6-9) potential with fitted parameters derived by Hagler et al. [14] for polar molecules. The relative contributions of these energies to the total interaction energies are small but occasionally different from Spackman et al.'s results [20], see table 11. Lattice

Table 8

Electrostatic properties of urea $\left(\times 10^{4}\right)$; net atomic charges $q(e)$, dipole moments $d(e \AA)$ and quadrupole moments $\mu\left(e \AA^{2}\right)$ are defined in a Cartesian molecular axial system with $z$ along the twofold axis $\mathrm{C} \rightarrow \mathrm{O}$, and $x$ along the normal to the molecular plane

\begin{tabular}{|c|c|c|c|c|c|c|c|c|c|c|}
\hline Atom & $q$ & $d_{x}$ & $d_{y}$ & $d_{z}$ & $\mu_{x x}$ & $\mu_{y y}$ & $\mu_{z z}$ & $\mu_{x y}$ & $\mu_{y z}$ & $\mu_{x z}$ \\
\hline $\mathrm{C} 1$ & 2522 & 0 & 0 & -509 & 1336 & -455 & -880 & 0 & 0 & 0 \\
\hline O1 & -4359 & 0 & 0 & -1113 & -136 & -346 & 482 & 0 & 0 & 0 \\
\hline N1 & -1670 & 0 & -33 & 80 & -1195 & 639 & 556 & 0 & 60 & 0 \\
\hline $\mathrm{H} 1$ & 1390 & 0 & 1014 & 505 & -227 & 123 & 104 & 0 & -16 & 0 \\
\hline $\mathrm{H} 2$ & 1198 & 0 & -71 & -1056 & -257 & 210 & 47 & 0 & -6 & 0 \\
\hline N1B & -1670 & 0 & 33 & 80 & -1195 & 639 & 556 & 0 & -60 & 0 \\
\hline $\mathrm{H} 1 \mathrm{~B}$ & 1390 & 0 & -1014 & 505 & -227 & 123 & 104 & 0 & 16 & 0 \\
\hline $\mathrm{H} 2 \mathrm{~B}$ & 1198 & 0 & 71 & -1056 & -257 & 210 & 47 & 0 & 6 & 0 \\
\hline
\end{tabular}


Table 9

Electrostatic interaction energies $(\mathrm{kJ} / \mathrm{mol})$ of $\mathrm{M}(1)$ in the urea structure with a number of neighbour molecules in the first two coordination spheres; the number of the molecules refers to fig. 11

\begin{tabular}{llrrrrrrr}
\hline $\begin{array}{l}\text { Bond } \\
\text { type }\end{array}$ & Neighbour & $E_{q-q}$ & $E_{q-d}$ & $E_{d-d}$ & $E_{q-\mu}$ & $E_{d-\mu}$ & $E_{\mu-\mu}$ & $E_{\text {tot }}$ \\
\hline a & $\mathrm{M}(1,001)$ & -18.99 & -22.01 & -5.69 & -1.84 & -0.72 & -0.28 & -49.53 \\
$\mathrm{~b}$ & $\mathrm{M}(2)$ & -13.76 & -12.76 & -2.21 & -1.08 & -0.32 & -0.07 & -30.21 \\
$\mathrm{c}$ & $\mathrm{M}(2,00 \overline{1})$ & +1.13 & -0.74 & +0.76 & -0.30 & +0.41 & -0.07 & +1.20 \\
$\mathrm{~d}$ & $\mathrm{M}(1,002)$ & -2.10 & -1.66 & -0.32 & -0.03 & 0.00 & 0.00 & -4.12 \\
$\mathrm{e}$ & $\mathrm{M}(1,010)$ & +3.56 & +1.10 & +0.07 & -0.33 & +0.04 & -0.04 & +4.39 \\
$\mathrm{f}$ & $\mathrm{M}(1,110)$ & +1.98 & +1.38 & +0.37 & +0.19 & +0.12 & -0.02 & +4.02 \\
$\mathrm{~g}$ & $\mathrm{M}(1, \overline{1} 10)$ & +1.50 & +0.94 & +0.18 & -0.04 & +0.02 & -0.01 & +2.58 \\
$\mathrm{~h}$ & $\mathrm{M}(2,001)$ & +2.49 & +1.47 & +0.20 & -0.04 & -0.01 & 0.00 & +4.12 \\
$\mathrm{i}$ & $\mathrm{M}(1,101)$ & -0.38 & +0.06 & +0.03 & +0.05 & -0.02 & +0.01 & -0.25 \\
\hline
\end{tabular}

energies were calculated by increasing the size of the crystal block around the reference molecule up to five cells in all crystallographic directions (containing 2001 molecules), corresponding with a summation limit of $25 \AA$. The calculated lattice energy of $-110 \mathrm{~kJ} / \mathrm{mol}$ compares well to the experimental value of -93 (6) $\mathrm{kJ} / \mathrm{mol}$, while Spackman et al. found $-66(24) \mathrm{kJ} / \mathrm{mol}$ for a small cluster of 32 molecules [20].

\subsection{Qualitative and quantitative $P B C$ analysis}

Hartman [21] performed a qualitative $\mathrm{PBC}$ analysis of the urea structure. The first coordination sphere of $\mathrm{M}(1)$ contains ten molecules, bound to $M(1)$ by three different types of bonds (table 11). As can be seen from fig. 11a, doubly hydrogen-bonded molecules are linked head-to-tail along the $c$-axis. This indicates a PBC running in the [001] direction consisting of type a bonds (table 12). Type $b$ bonds are singly hydrogenbonded. From table 10 it appears that the corresponding bond energy is indeed about half as large as for bond a. It also appears from table 10 that type $\mathrm{c}$ bonds are weak Van der Waals bonds. Therefore it seems appropriate not to consider these as "strong bonds" in the context of the PBC theory (as was assumed by Hartman [21]). In a projection along the $c$-axis (fig. 11b) it appears that bonds of type $b$ form a PBC in the $[1 \overline{1} 0]$

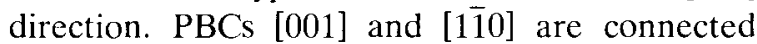
within $d_{110}$. In a projection along [1 $\left.1 \overline{1} 0\right]$ in fig. $11 \mathrm{c}$, PBCs [110] are mutually linked in the F-slice $d_{001}$ by PBCs [110]. If bond $\mathrm{c}$ is considered as a strong bond, also $d_{111}$ is an F-slice by means of connection with PBCs [101] consisting of $b$ and $c$ type

Table 10

Total interaction energies $E(\mathrm{tot})(\mathrm{kJ} / \mathrm{mol})$ for urea as the sum of Coulomb energies $E(\mathrm{Coul})(\mathrm{kJ} / \mathrm{mol})$ and Van der Waals energies $E(\mathrm{vdW})(\mathrm{kJ} / \mathrm{mol})$, as found by Spackman et al. [20] and by us; the numbering of the molecules refers to fig. 11

\begin{tabular}{|c|c|c|c|c|c|c|c|}
\hline \multirow{2}{*}{$\begin{array}{l}\text { Bond } \\
\text { type }\end{array}$} & \multirow[t]{2}{*}{ Neighbour } & \multicolumn{3}{|c|}{ Spackman et al. } & \multicolumn{3}{|c|}{ This paper } \\
\hline & & $E($ Coul $)$ & $E(\mathrm{vdW})$ & $E($ tot $)$ & $E($ Coul $)$ & $E(\mathrm{vdW})$ & $E($ tot $)$ \\
\hline $\mathrm{a}$ & $\mathrm{M}(1,001)$ & $-50(14)$ & 7 & -43 & -49.53 & $4 . \overline{46}$ & -45.08 \\
\hline b & $\mathrm{M}(2)$ & $-32(10)$ & 7 & -25 & -30.21 & -2.11 & -32.32 \\
\hline $\mathrm{c}$ & $M(2,00 \overline{1})$ & $1(8)$ & 6 & 7 & 1.20 & -2.51 & -1.32 \\
\hline d & $\mathrm{M}(1,002)$ & $-5 \quad(3)$ & 0 & -5 & -4.12 & -0.16 & -4.28 \\
\hline $\mathrm{e}$ & $\mathrm{M}(1,010)$ & $4(2)$ & -2 & 2 & 4.39 & -2.35 & 2.04 \\
\hline $\mathrm{f}$ & $\mathrm{M}(1,110)$ & 7 (2) & 0 & 7 & 4.02 & -0.49 & 3.54 \\
\hline $\mathrm{g}$ & $\mathbf{M}(1, \overline{1} 10)$ & 4 (1) & 0 & 4 & 2.58 & -0.28 & 2.31 \\
\hline $\mathrm{h}$ & $\mathrm{M}(2,001)$ & $5(4)$ & 0 & 5 & 4.12 & -0.59 & 3.53 \\
\hline $\mathrm{i}$ & $\mathrm{M}(1,101)$ & -1 (3) & 0 & -1 & -0.25 & -0.64 & -0.89 \\
\hline
\end{tabular}


406

E.S. Bock et al. / From wace function to crystal morphology
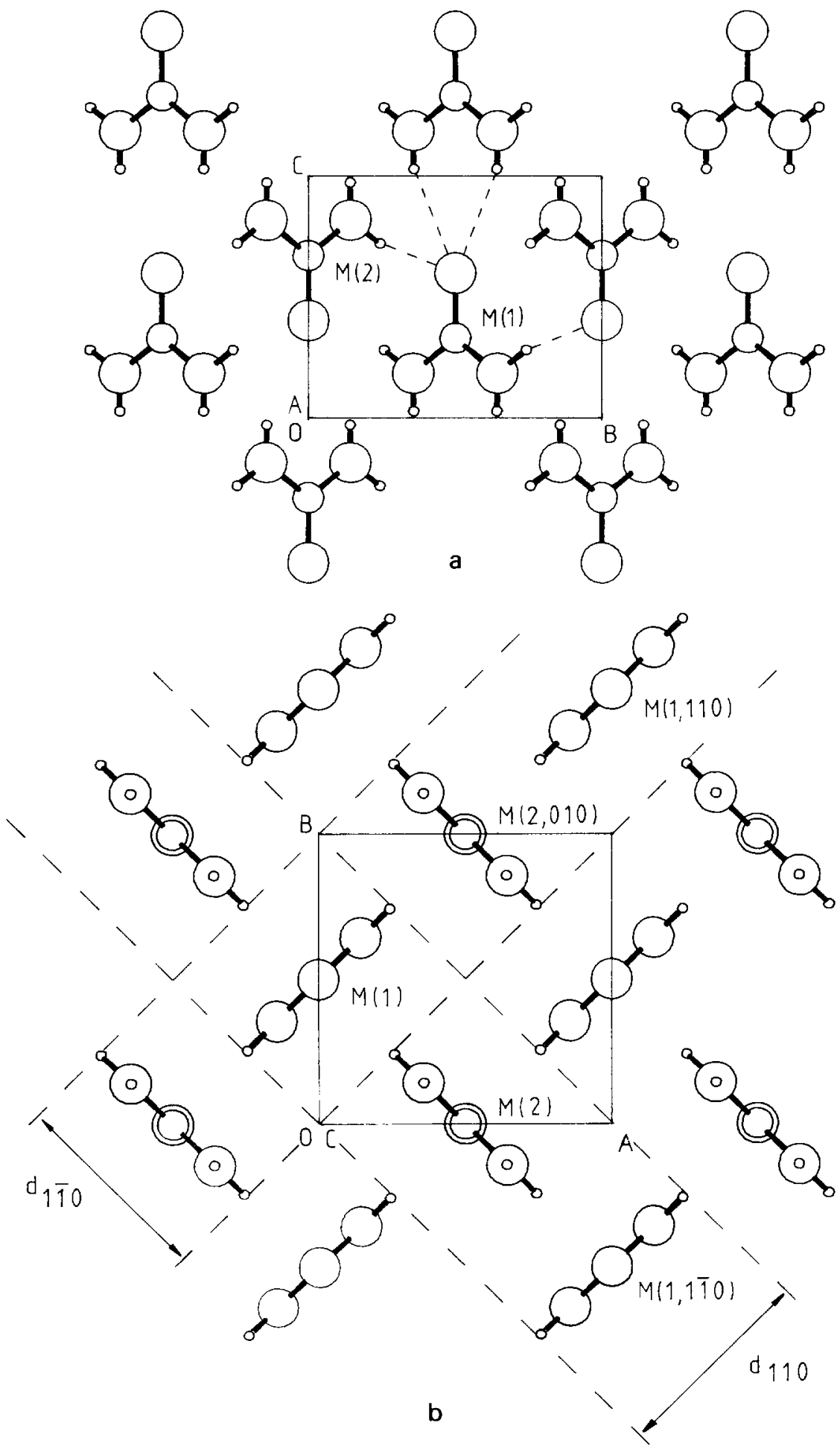


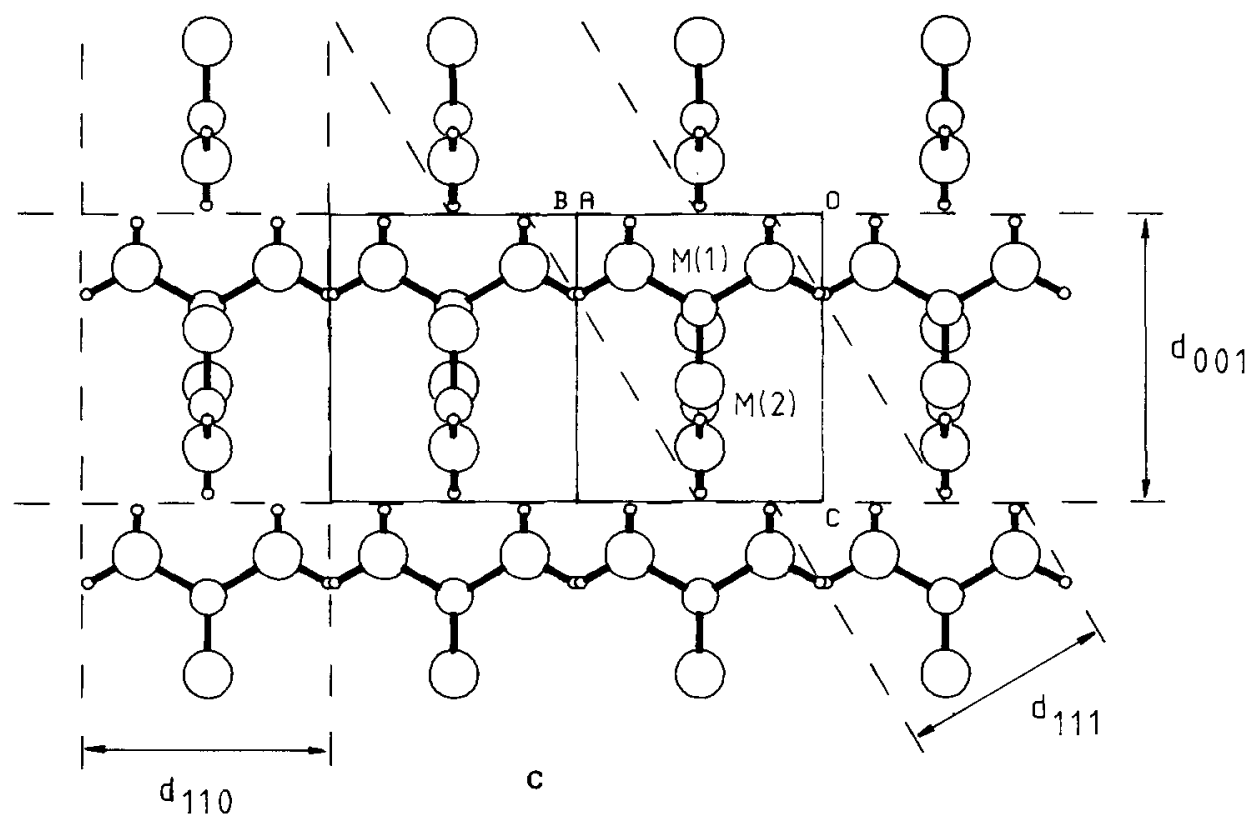

Fig. 11. (a) Projection of the urea crystal structure along the $a$-axis. (b) Projection of the urea crystal structure along the $c$-axis. (c) Projection of the urea crystal structure along [110].

bonds. PBCs are shown in table 12. Attachment energies calculated with MOLINT are listed in table 13. The theoretical growth form showing the prism (110) and (001) is shown in fig. 14a. Again, attachment energy calculations based on nearest neighbours only yield similar results.

\subsection{Critical Ising temperatures of connected nets}

Critical Ising temperatures $\theta_{h k l}^{\mathrm{c}}$ were calculated for the three different connected nets by means of the computer program TC [8]. In fig. 12 the connected nets for the urea structure, drawn with BEHNG2 [25], are presented. Rectangularization of the nets is shown in fig. 13. The calcu-

Table 11

Nearest neighbours of $\mathrm{M}(1)$ in the urea structure

\begin{tabular}{llll}
\hline $\begin{array}{l}\text { Bond } \\
\text { type }\end{array}$ & Bond & Contact & $\begin{array}{l}\text { Short } \\
\text { distance } \\
(\AA)\end{array}$ \\
\hline a & $\mathrm{M}(1)-\mathrm{M}(1,001)$ & Double $\mathrm{N}-\mathrm{H} \cdots$ O bond & 2.994 \\
$\mathrm{~b}$ & $\mathrm{M}(1)-\mathrm{M}(2)$ & Single $\mathrm{N}-\mathrm{H} \cdots$ O bond & 3.033 \\
$\mathrm{c}$ & $\mathrm{M}(1)-\mathrm{M}(2,00 \overline{1})$ & Van der Waals bond & 3.526 \\
\hline
\end{tabular}

lated values for $\theta^{c}$, using the ratios of the nearest neighbour bond strengths ( $\mathrm{a}, \mathrm{b}$ and $\mathrm{c}$ in table 10 ), are presented in table 14 . The resulting theoretical growth form is the same as for the $E_{\text {att }}$ model drawn in fig. 14a.

\subsection{Equilibrium and Donnay-Harker morphology}

The equilibrium form based on relative surface energies $\gamma_{h k l}$ proportional to $E_{h k l}^{\text {att }} d_{h k l}$ (table 14) is constructed in fig. 14b. This shows also the hemihedral (111) face. An ad hoc assumption was made by suppressing the appearance of (1111). The Donnay-Harker morphology resulting from slice thickness data (table 14) is given in fig. 14c.

\subsection{Comparison of theoretical and experimental morphologies}

From a mixture of water and ethanol, urea crystallizes as long thin prisms $\{110\}$ bounded by $\{111\}$ or $\{001\}$, from water and benzene as short prisms $(\{110\}$ and $\{111\})$ and sometimes cubelike ( $\{110\}$ and $\{001\})$ [22]. It appears that the theoretical growth form of urea compares quite well to 
Table 12

Schematic configuration of the PBCs in urea

\begin{tabular}{lll}
\hline PBC & Constituting molecules & Bonds \\
\hline$[001]$ & $\mathrm{M}(1)-\mathrm{M}(1,001)$ & $\mathrm{a}$ \\
{$[1 \overline{1} 0]$} & $\mathrm{M}(1)-\mathrm{M}(2)-\mathrm{M}(1,1 \overline{1} 0)$ & $2 \mathrm{~b}$ \\
{$[101]$} & $\mathrm{M}(1)-\mathrm{M}(2)-\mathrm{M}(1,101)$ & $\mathrm{b}+\mathrm{c}$ \\
\hline
\end{tabular}

crystals grown from ethanol and benzene. Urea crystals grown by sublimation are elongated in the [001] direction, showing $\{110\}$ and $\{001\}$ [27]. The Donnay-Harker form shows more faces than those constructed from the Ising or $E_{\text {att }}$ model. Experimentally, these extra $\{101\}$ forms were never observed, so it may be concluded that the attachment energy and Ising models give a better fit to the observation.

The influence of solvent adsorption on the crystal habit is quite complex in the case of urea: solvent-growth experiments as mentioned above indicate that water retards the growth of $\{110\}$ in a much stronger way than less polar solvents like

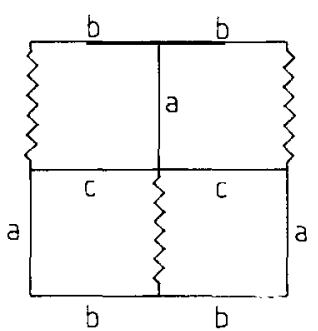

a

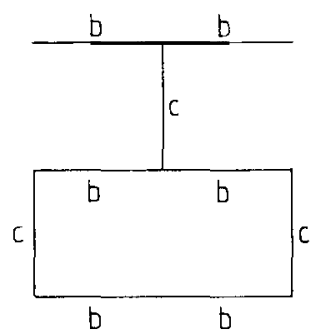

b
Fig. 13. (a) Rectangularized connected net of (110); (b) rectangularized connected net of (111).

ethanol-benzene. At first sight, however, solvent adsorption on $\{001\}$ should be strong, because the dipole moments of the urea molecules are parallel to the normal on the face, while for $\{110\}$ the molecular dipole moments are perpendicular to the face normal. This indicates growth retardation of $\{001\}$ which appears not to be found experimentally. MD simulations of these inter-

Table 13

Attachment energies for urea for nearest neighbour bonds and in the summation limit of $30 \AA$

\begin{tabular}{|c|c|c|c|c|}
\hline F-face & $\begin{array}{l}\text { Nearest neighbour } \\
\text { bonds per molecule }\end{array}$ & $\begin{array}{l}E_{h i k l}^{\mathrm{atl}}(\mathrm{Coulomb}) \\
(\mathrm{kJ} / \mathrm{mol})\end{array}$ & $\begin{array}{l}E_{h k l}^{\mathrm{all}}(\mathrm{vdWaals}) \\
(\mathrm{kJ} / \mathrm{mol})\end{array}$ & $\begin{array}{l}E_{h / k l}^{\mathrm{atl}}(\mathrm{total}) \\
(\mathrm{kJ} / \mathrm{mol})\end{array}$ \\
\hline$(110)$ & $b+c$ & -19.27 & -16.65 & -35.92 \\
\hline$(001)$ & $a+2 c$ & -40.50 & -8.10 & -48.60 \\
\hline (111) & $(2 a+2 b+3 c) / 2$ & -64.71 & -13.07 & -77.78 \\
\hline \multicolumn{5}{|l|}{$E_{c r}($ total $)$} \\
\hline$(\mathrm{kJ} / \mathrm{mol})$ & $a+2 b+2 c$ & -90.75 & -18.80 & -109.55 \\
\hline
\end{tabular}

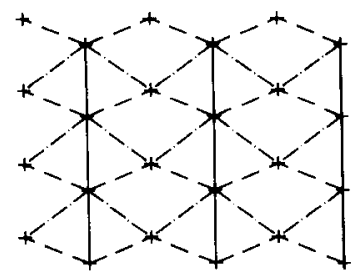

a

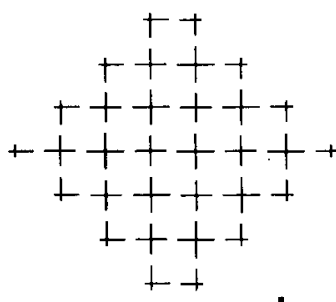

b

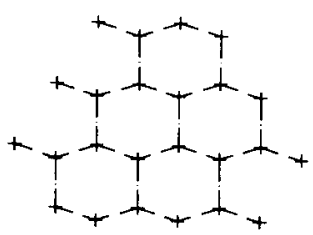

C

Fig. 12. Projections of urea crystal slices along their normal: (a) connected net of (110), rectangularized in fig. 13a; (b) rectangular connected net of $(001)$; (c) triangular connected net of (111), rectangularized in fig. $13 \mathrm{~b}$. 

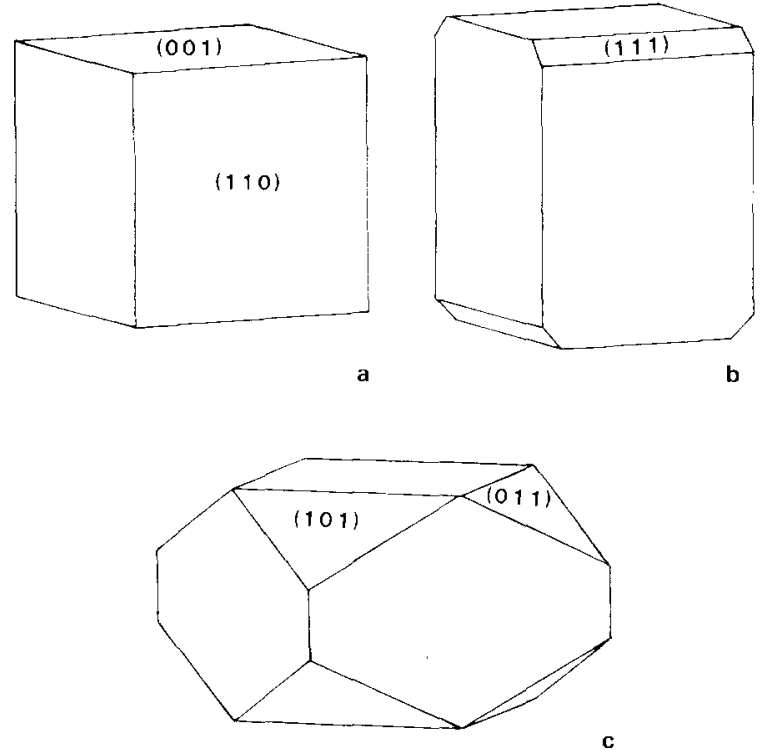

Fig. 14. Theoretical habits of urea: (a) Growth form according to $E_{\text {att }}$ and Ising models; (b) equilibrium form; (c) growth form according to the Donnay-Harker model.

faces are in progress now to investigate adsorption effects on the morphology.

\section{Conclusions and discussion}

Accurate intermolecular energies have been calculated based on ab initio quantum-chemical methods. For urea these compare very well to energies derived from $\mathrm{X}$-ray diffraction. In the case of $\alpha$-glycine the differences are larger. For both compounds the F-faces were determined according to the PBC theory. In the case of

Table 14

Connectedness (c) or non-connectedness (nc) of urea nets, critical Ising temperatures $\theta_{h k l}^{c}, 1 / d_{h k l}$ values and relative specific surface energies

\begin{tabular}{lllll}
\hline Face & Connectedness & $\left(\theta_{h k l}^{\mathrm{c}}\right)^{-1}$ & $\begin{array}{l}1 / d_{h k l} \\
\left(\AA^{-1}\right)\end{array}$ & $\gamma_{h k l}$ \\
\hline$(110)$ & $\mathrm{c}$ & 0.505 & 0.250 & 0.400 \\
$(001)$ & $\mathrm{c}$ & 0.615 & 0.212 & 0.641 \\
$(111)$ & $\mathrm{c}$ & 2.356 & 0.328 & 0.659 \\
$(101)$ & $\mathrm{nc}$ & - & 0.276 & - \\
$(200)$ & $\mathrm{nc}$ & - & 0.353 & - \\
\hline
\end{tabular}

$\alpha$-glycine the calculation of attachment energies without performing a structural PBC analysis results in a growth form showing predominantly F-faces. This theoretical growth form is in good agreement with crystals grown from aqueous solution. The experimental sublimation form, however, seems to show some pseudo F-faces. It appears that the morphology resulting from first neighbour interaction models (Ising and attachment energy) is very similar to the one based on attachment energy calculations in the electrostatic summation limit. This is partly due to the poor morphology of the investigated crystals.

\section{Acknowledgements}

The authors acknowledge the financial support of the Netherlands Organization for Scientific Research (NWO/SON). The authors wish to thank Professor P. Hartman for valuable advise and Dr. L.J.P. Vogels for help with the program BEHNG2 V.2.1 [25].

\section{References}

[1] J.D.H. Donnay and D. Harker, Am. Mineralogist 22 (1937) 446

[2] P. Hartman and W.G. Perdok, Acta Cryst. 8 (1955) 49, 521.

[3] P. Hartman, in: Crystal Growth: An Introduction, Ed. P. Hartman (North-Holland, Amsterdam, 1973) p. 367.

[4] P. Hartman and P. Bennema, J. Crystal Growth 49 (1980) 145.

[5] Z. Berkovitch-Yellin, J. Am. Chem. Soc. 107 (1985) 8239.

[6] J.L. Derissen, P.H. Smit and J. Voogd, J. Phys. Chem. 81 (1977) 1474

[7] W.K. Burton, N. Cabrera and F.C. Frank, Phil. Trans. Roy. Soc. London A 243 (1951) 299.

[8] J.J.M. Rijpkema, H.J.F. Knops, P. Bennema and J.P. van der Eerden, J. Crystal Growth 61 (1982) 295.

[9] P. Bennema, L. Xiang Yang, K. Lewtas, R.D. Tack, J.J.M. Rijpkema and K.J. Roberts, to be published.

[10] P. Hartman, J. Crystal Growth 49 (1980) 157.

[11] A. Szabo and N.S. Ostlund, Modern Quantum Chemistry (Macmillan, New York, 1982) p. 151.

[12] F.L. Hirshfeld, Theoret. Chim. Acta 44 (1977) 129.

[13] A.D. Buckingham, in: Intermolecular Interactions: From Diatomics to Biopolymers, Ed. B. Pullman (Wiley, New York, 1978) p. 1 
[14] A.T. Hagler, E. Huler and S. Lifson, J. Am. Chem. Soc. 96 (1976) 5319.

[15] J.P. Legros and A. Kvick, Acta Cryst. B 36 (1980) 3052.

[16] International Tables of Crystallography, Vol. A (Reidel, Dordrecht, 1983).

[17] J. Voogd, J.L. Derissen and F.B. van Duyneveldt, J. Am. Chem. Soc. 103 (1981) 7701.

[18] J. Voogd, Thesis, Rijksuniversiteit Utrecht (1982) ch. 4.

[19] J.E. Worsham, Jr., H.A. Levy and S.W. Peterson, Acta Cryst. 10 (1957) 319.

[20] M.A. Spackman, H.P. Weber and B.M. Craven, J. Am. Chem. Soc. 110 (1988) 775.

[21] P. Hartman, in: Adsorption et Croissance Cristalline, Colloq. Intern. CNRS, No. 152 (CNRS, Paris, 1965) p 477.
[22] P. Groth, Chemische Krystallographie, Vol. 3 (Engelmann, Leipzig, 1910) p. 539.

[23] M. Eisenstein, Acta Cryst. B 44 (1988) 412.

[24] M. Dupuis, D. Spangler and J.J. Wendoloski, NRCC Software Catalog, Vol. 1, Program N.QG01 (GAMESS), 1980 ;

M.F. Guest and J. Kendrick, GAMESS Users Manual, Daresbury Laboratory, 1985.

[25] L.J.P. Vogels and H. Meekes, Program BEHNG2 V2.1, unpublished.

[26] E. Dowty, SHAPE, V3.0, 1988.

[27] R.S. Feigelson, R.K. Route and T.-M. Kao, J. Crystal Growth 72 (1985) 585. 